ARTICLE

Received 18 Mar 2014 | Accepted 9 Sep 2014 | Published 28 Oct $2014 \quad$ DOl: 10.1038/ncomms6217

\title{
RNAi-based functional selection identifies novel cell migration determinants dependent on PI3K and AKT pathways
}

Minchul Seo ${ }^{1,2, \star}$, Shinrye Lee $e^{1,3, \star}$, Jong-Heon Kim¹, Won-Ha Lee ${ }^{4}$, Guang Hu${ }^{5}$, Stephen J. Elledge ${ }^{6}$ $\&$ Kyoungho Suk ${ }^{1}$

Lentiviral short hairpin RNA (shRNA)-mediated genetic screening is a powerful tool for identifying loss-of-function phenotype in mammalian cells. Here, we report the identification of 91 cell migration-regulating genes using unbiased genome-wide functional genetic selection. Individual knockdown or cDNA overexpression of a set of 10 candidates reveals that most of these cell migration determinants are strongly dependent on the PI3K/PTEN/ AKT pathway and on their downstream signals, such as FOXO1 and p70S6K1. ALK, one of the cell migration promoting genes, uniquely uses $\mathrm{p} 55 \gamma$ regulatory subunit of $\mathrm{PI} 3 \mathrm{~K}$, rather than more common p85 subunit, to trigger the activation of the PI3K-AKT pathway. Our method enables the rapid and cost-effective genome-wide selection of cell migration regulators. Our results emphasize the importance of the PI3K/PTEN/AKT pathway as a point of convergence for multiple regulators of cell migration.

\footnotetext{
${ }^{1}$ Department of Pharmacology, Brain Science \& Engineering Institute, BK21 Plus KNU Biomedical Convergence Program, Kyungpook National University School of Medicine, Daegu, Republic of Korea. ${ }^{2}$ College of Medicine, Dongguk University, Gyeongju, Republic of Korea. ${ }^{3}$ Korea Brain Research Institute (KBRI), Daegu, Republic of Korea. ${ }^{4}$ KNU Creative BioResearch Group, School of Life Sciences and Biotechnology, Kyungpook National University, Daegu, Republic of Korea. ${ }^{5}$ Laboratory of Molecular Carcinogenesis, National Institute of Environmental Health and Sciences, Research Triangle Park, North Carolina 27709, USA. ${ }^{6}$ Department of Genetics, Howard Hughes Medical Institute, Division of Genetics, Brigham and Women's Hospital, Harvard Medical School, Boston, Massachusetts 02115, USA. * These authors contributed equally to this work. Correspondence and requests for materials should be addressed to K.S. (email: ksuk@knu.ac.kr).
} 
C ell migration is a dynamic process that requires coordinated cytoskeletal regulation and proper polarization, and is governed by the extracellular microenvironment, such as chemokines and growth factors. Cell migration is central to development, wound repair and tissue remodelling, and plays a major role in cancer metastasis ${ }^{1,2}$. Cell migration to specific sites of inflammation or infection is also essential for immune system function, with respect to the elimination of foreign or infectious agents $^{3}$. Given the relevance of cell migration in a variety of physiological and pathological conditions, we attempted to identify novel genes that regulate cell migration using the short hairpin RNA (shRNA)-based functional selection of cell migration phenotypes. Lentivirally delivered shRNAs were used to produce stable transcript knockdown in mouse fibroblast cells and to conduct loss of function genetic selections.

Genetic screening for genes that regulate cell migration and morphology has been previously performed in various invertebrate model organisms, such as, Drosophila melanogaster and Caenorhabditis elegans ${ }^{4,5}$. The development of small interfering RNA (siRNA) and shRNA technology has also made it feasible to perform genetic screening in mammalian cells. siRNAs can be generated in organisms using shRNAs, consisting of a sequence of 21-29 nt, a short loop region, and the reverse complement of the 21-29 nt region ${ }^{6}$. shRNA libraries have been used to perform genetic screens in tissue culture cells for a variety of phenotypes ${ }^{7}$. Recent studies have analysed the closures of scratches in cellular monolayers after growth factor stimulation, using siRNA library targeting kinase and phosphatase genes ${ }^{8,9}$. siRNA-based screening was performed to identify regulators of multiple cell adhesion complex formation ${ }^{10}$, and RNAi screening to identify inhibitors of cell migration using SKOV-3 cells (a highly motile ovarian carcinoma cell line) ${ }^{11}$. More recently, new regulators of morphology, cytoskeletal organization and cell migration in human cells have been identified using genome-wide RNAi morphology screening data in D. melanogaster cells ${ }^{12}$. Pooled shRNAs were also used for the genome-wide screen of cell migration regulators ${ }^{13}$, and, in that study, barcode microarray analysis was used to identify enriched shRNAs.

Herein, we adopted a selection and sequencing strategy to identify both cell migration-accelerating and -impairing genes using a genome-wide pooled shRNA library. Selection was performed using Boyden chamber assays followed by the separation and enrichment of cells with increased or decreased motility. shRNAs were then retrieved from selected cells and directly identified by half-hairpin barcode sequencing. This selection process resulted in the identification of 91 positive or negative regulators of cell migration; 29 of which genes had not been previously reported as cell migration regulators by RNAi screening. A set of 10 shRNAs was chosen for further validation studies, and these revealed remarkable dependences on the phosphoinositide-3 kinase (PI3K)/phosphatase and tensin homologue (PTEN)/AKT signalling pathway for cell migration acceleration or impairment.

\section{Results}

Genome-wide functional selection of cell migration regulators. To identify novel cell migration-regulating genes, RNAi-based functional selection was performed. After introducing 63,996 pooled lentiviral mouse shRNAs targeting 21,332 genes into NIH3T3 mouse fibroblast cells, the shRNAs that accelerated or impaired baseline motility were selected using the transwell migration assay (Fig. 1a). Pooled recombinant lentivirus expressing shRNAs was generated by transfecting HEK293T cells with pHAGE-mir30-RFP-shRNA (targeting the mouse genome) (Fig. 1b), pVSV-G, pTat, $p P M 2$ and $p$ Rev. NIH3T3 fibroblast cells were infected with the 63,996 pooled lentiviral mouse shRNA library at a multiplicity of infection (m.o.i.) of one ${ }^{14,15}$. Two days after infection, shRNA-infected cells were selected with puromycin, placed into the upper compartment of a transwell unit and allowed to migrate through the perforated membrane to the lower compartment. Cells that exhibited accelerated or impaired migration were isolated from lower or upper compartments after 5 or $24 \mathrm{~h}$ of incubation, respectively; 5 and $24 \mathrm{~h}$ were chosen as optimal incubation times for these purposes (Supplementary Fig. 1). In fact, different cell types and assay conditions were used in the previous migration screens. These discrepancies in the experimental setups may determine the degree of agreement of the current results with published data (Supplementary Table 1). Cells with the desired phenotypes were enriched by repeating the procedure five times. After enrichment, genomic DNA was isolated, and shRNAs integrated into chromosomes were retrieved by PCR amplification, cloned and sequenced. The determined sequences of half-hairpin barcode were used to identify the shRNAs. This selection method identified 91 shRNAs that accelerated or impaired migration (Tables 1 and 2). Sixty-two of the 91 identified target genes have been previously associated with cell migration by RNAi screen $^{8-10,13,16}$ (Supplementary Tables 2 and 3). The remaining 29 target genes had not been previously identified as cell migration regulators by RNAi screen. To determine relationships between cell migration regulators, a signalling network was built by ingenuity pathway analysis (IPA) analysis (Fig. 2). This analysis revealed that the newly identified cell migration regulators were closely linked to various cell movement signalling components (Fig. 2a). The 91 cell migration-regulating genes identified were mapped to several major biological functions by IPA analysis; these functions included cell movement and morphology (41\%), cellular assembly and organization (21\%), cell-to-cell signalling (20\%), and protein trafficking and molecular transport (18\%) (Fig. 2b). Functional sub-networks were also constructed based on the biological functions in which they participate (Supplementary Fig. 2a-d). Sub-network topology indicated a close interaction between the newly identified cell migration regulators and various functional components. Direct or indirect protein-protein interactions were also found between the identified regulators and other cell migration signalling components (Supplementary Fig. 2e). Several additional targets were identified, not found in the primary selection, which might be predicted to modulate cell migration through network inference; these include CTNNB1, CDK4, MYC, IRS4, EIF3F, KIF5C and so on. In addition, a partial cell migration network was constructed, based on signalling pathways initiated by growth factor/receptor tyrosine kinase, fibronectin/ integrin or chemotactic factor/GPCR (Supplementary Fig. 2f). Many nodes in the network were candidate genes identified in the current selection. PI3K, PTEN and AKT emerged as convergent points in multiple networks. This result was not surprising, as the $\mathrm{PI} 3 \mathrm{~K} / \mathrm{AKT}$ axis is known to regulate cell migration under diverse conditions. In addition, subcellular localization and disease association of the cell migration-regulating genes were analysed (Supplementary Fig. 3 and Supplementary Tables 4 and 5). The majority of the genes were associated with cancer and neurological diseases.

Validation of selected cell migration regulators. Among the 91 shRNAs identified (Tables 1 and 2), 10 were selected for further investigation (Supplementary Table 6). These shRNAs were selected, as little or no evidence linked the corresponding target genes with the regulation of cell migration. shRNA-induced phenotypes were validated using siRNA-mediated knockdown. 
a
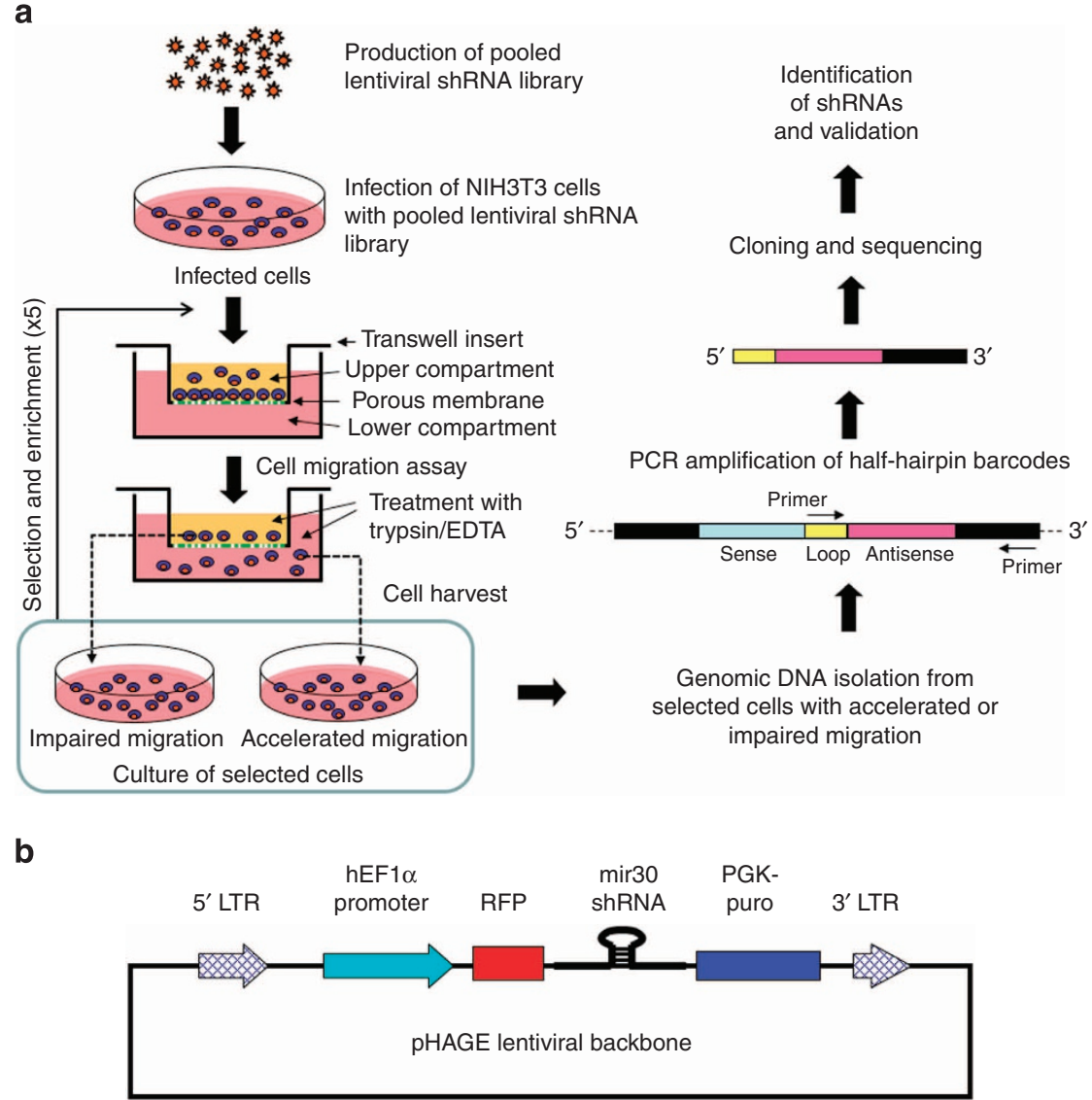

Figure 1 | Schematic representation of the RNAi-based selection of cell migration regulators. (a) Overview of the selection procedure. The production and infection of genome-wide lentiviral shRNA library are described in Methods. Two days after lentiviral infection, NIH3T3 mouse fibroblast cells were seeded onto transwell inserts and allowed to migrate across the porous membrane at $37^{\circ} \mathrm{C}$ for 5 or $24 \mathrm{~h}$, to select cells with an increased or decreased migration phenotype, respectively. Migrated or non-migrated cells were collected by trypsin-EDTA treatment from the lower or upper faces, respectively, of inserts, and reseeded onto transwell culture inserts for a second round of selection (this process was repeated five times). After the final round of selection, shRNAs were retrieved by PCR from selected cells and identified by sequencing. (b) Diagram of pHAGE-mir30-RFP-shRNA vector. The pooled lentiviral vector contained 63,996 different mir30-based shRNAs targeting 21,332 mouse genes.

To avoid off-target effects, five siRNA duplexes were synthesized for each shRNA candidate identified. Targeting sequences of the siRNAs were distinct from those of the shRNA constructs. The wound-healing assay revealed that transfection with the siRNAs of five migration-accelerating shRNA candidates (mtmr1, lats2, dock3, myo5a and ptpn14) (Fig. 3a,b) or with the siRNAs of five migration-impairing shRNA candidates (csnk2a2, arid4a, ppp3cc, irf4 and alk) (Fig. 3c,d) increased or decreased, respectively, the motility of NIH3T3 fibroblast cells as compared with control siRNA transfectant. The cell migration-regulating activities of these candidates were also examined using the transwell migration assay, and similar results were obtained (Fig. 4a,b). In addition, the cell migration-regulating activities of these candidates were confirmed in L929 mouse fibroblast and mouse embryonic fibroblast (MEF) cells using the transwell migration assay, which indicated that the observed cell migration-regulating effects of the siRNAs were not limited to a single cell line (Fig. 4c,d). siRNA-mediated knockdown of the 10 target genes mentioned above was confirmed by RT-PCR (Fig. 3b,d), and $>50 \%$ knockdown was achieved for all siRNAs tested. siRNAmediated knockdown of the target genes was also confirmed at the protein levels by western blot analysis (Supplementary Fig. 4a-e). Furthermore, it should be noted that the cell migration-regulating properties of siRNAs in the wound-healing assay and transwell migration assay were not due to effects on cell proliferation, as determined by MTT assay (Supplementary Fig. $4 \mathrm{f}-\mathrm{h})$. Validation experiments were extended to other hits identified in the selection and network-derived candidates. Additional 36 hits (20 migration-accelerating shRNAs and 16 migration-impairing shRNAs) and four network-derived candidates (ctnnb1, cdk4, myc and irs4) were tested using pooled retroviral shRNAs (Supplementary Table 7, Supplementary Fig. 5 and Supplementary Movies 1-3). As a result, 71.7\% (33/46) of the hits showed cell migration-regulating activity consistent with the primary screen. Among the four network-derived candidates, ctnnbl and $c d k 4$ shRNAs promoted and inhibited cell migration, respectively, thereby demonstrating 50\% validation for the network analysis.

Common roles of the PI3K/PTEN/AKT pathways. Network analysis of the cell migration regulators identified indicated that the PI3K/PTEN/AKT signalling pathway plays a central role (Fig. 2 and Supplementary Fig. 2). PI3K/PTEN/AKT signalling has been previously associated with cell migration. For example, $\mathrm{PI} 3 \mathrm{~K} / \mathrm{AKT}$ has been reported to enhance actin remodelling, to generate membrane protrusions and to induce cell migration and cell invasion via remodelling of the actin cytoskeleton ${ }^{17}$. PTEN is a lipid phosphatase that dephosphorylates the D3 position of phosphatidylinositol-3,4,5-trisphosphate (PIP3), a 
Table 1 | List of shRNAs that accelerated cell migration (with targets genes inhibiting cell migration).

\section{Symbols}

Target genes

\section{Cell cycle}

dgkz

Cell morphology

dock3

myo5a

Cell signalling

ppid

Cellular development

acvr1

cdk13

Inflammatory response and disease

enpp2

gfra2

icam2

lats2

Inflammatory response and disease

ptpn14

h2-Q10

Molecular transport

pcytia

pts

Post-translational modification

sbf2

Other/unknown

A930006J02Rik

atmin

cntnap4

corolb

D630033011Rik

gabra4

gm379

gm1971

gm5615

gm12273

gnal

gpkow

itprip/2

lair1

LOC668961

mia3

mpc1

mtmr1

otud6b

ptpn3

ptpn23

ptx4

rfpl4

trim59

trip10

usp 45

zbed3

Myosin VA

Pentraxin 4
Diacylglycerol kinase zeta

Dedicator of cyto-kinesis 3

Peptidylprolyl isomerase D (cyclophilin D)

Activin A receptor, type 1

Cyclin-dependent kinase 13

Ectonucleotide pyrophosphatase/phosphodiesterase 2

Glial cell line derived neurotrophic factor family receptor alpha 2

Intercellular adhesion molecule 2

Large tumour suppressor 2

Protein tyrosine phosphatase, non-receptor type 14

Histocompatibility 2, Q region locus 10

Phosphate cytidylyltransferase 1, choline, alpha isoform

6-pyruvoyl-tetrahydropterin synthase

SET-binding factor 2

RIKEN cDNA A930006J02 gene

ATM interactor

Contactin-associated protein-like 4

Coronin, actin-binding protein $1 \mathrm{~B}$

RIKEN cDNA D630033011 gene

Gamma-aminobutyric acid A receptor, subunit alpha 4

Gm379-predicted gene 379

Gm1971-predicted gene 1971

Predicted gene 5615

Gm12273 predicted gene 12273

Guanine nucleotide-binding protein, alpha stimulating, olfactory type

$\mathrm{G}$ patch domain and KOW motifs

Inositol 1,4,5-triphosphate receptor-interacting protein-like 2

Leukocyte-associated Ig-like receptor 1

LOC668961 spindlin 2 family member

Melanoma inhibitory activity 3

Mitochondrial pyruvate carrier 1

Myotubularin related protein 1

OTU domain containing 6B

Protein tyrosine phosphatase, non-receptor type 3

Protein tyrosine phosphatase non-receptor type 23

Ret finger protein-like 4

Tripartite motif-containing 59

Thyroid hormone receptor interactor 10

Ubiquitin specific peptidase 45

Zinc-finger, BED domain containing 3
NM_177324

GenBank accession No.

NM_138306

NM_153413

NM_010864

NM_026352

NM_007394

NM_027118

NM_001136077

NM_008115

NM_010494

NM_015771

NM_008976

BC042572

NM_009981

NM_011220

AK020818

NM_177700

NM_130457

NM_011778

XM_001001707

NM_010251

XM_142052

XM_001472879

NM_001033783

XM_001479118

NM_010307

NM_173747

NM_001033380

NM_001113474

XM_001006595

NM_177389

NM_018819

NM_016985

NM_152812

NM_011207

NM_001081043

NM_001163416

NM_138954

NM_025863

NM_134125

NM_152825

NM_028106

shRNAs were categorized by biological function using DAVID (database for annotation, visualization and integrated discovery).

second messenger produced by PI3K and that activates $\mathrm{AKT}^{18,19}$. PTEN is also known to antagonize the cell migration-promoting activity of PI3K. To determine whether the PI3K/PTEN/AKT signalling pathway is involved in the accelerated or impaired migration induced by shRNAs, we first assessed AKT phosphorylation after knocking down dock3, mtmr1, ptpn14, lats 2 and $m y o 5 a$, and after overexpressing alk and irf4. A knockdown of dock3, mtmr1, ptpn14 or $m y o 5 a$, but not of lats2, or the overexpression of alk or irf4 induced the phosphorylation of AKT (Fig. 5a-c). The overexpression of alk or irf4 in transfectants was confirmed by western blot analysis (Fig. 5d). In addition, we used pharmacological inhibitors of PI3K or AKT to evaluate the role of PI3K/AKT signalling in the accelerated or impaired cell migration by shRNAs. siRNA-mediated knockdown was done to upregulate cell migration for cell migrationinhibiting genes, whereas cDNA overexpression was done to upregulate cell migration for cell migration-promoting genes. The accelerated cell migration observed after mtmr1, dock3, myo5a or ptpn14 (but not lats2) knockdown was significantly attenuated by AKT or PI3K inhibitors in the wound-healing (Fig. 6a) and transwell assays (Fig. 7a). Similarly, the accelerated cell migration observed for alk or irf4 overexpression was also attenuated by these inhibitors in the wound-healing (Fig. 6b) and transwell assays (Fig. 7b). Taken together, these results indicate that the cell 
Table 2 | List of shRNAs that impaired cell migration (with targets genes promoting cell migration).

\section{Symbols}

Target genes

\section{Cell cycle}

clip1

Cell morphology

actg1

add1

akt3

des

itgb8

pik3ca

$r d x$

sep15

sorbs 1

tpm3

vim

Cell signalling

apc

Cellular

adam2

Cellular development

alk

irf4

igftr

Cellular movement

abl1

$a b / 2$

arhgap5

csnkla1

csnk2a2

irgm1

itgav

nrp2

pak4

Metabolism

abi2

mogat1

Other/unknown

akap11

arhgap26

arid4a

ccdc34

cdc42

cdh12

cmtm2b

defb20

eif3e

frmd6

g3bp2

gpr143

hpdl

hsd17b11

pdgfrl

ppp3cc

rnf139

rp17

stradb

svil

tm4sf5

Desmin

Radixin

Vimentin

ganization

Neuropilin 2

Supervillin
CAP-GLY domain containing linker protein 1

Actin, gamma, cytoplasmic 1

Adducin 1 (alpha)

V-akt murine thymoma viral oncogene homologue 3 (protein kinase B, gamma)

Integrin beta 8

Phosphoinositide-3-kinase, catalytic, alpha polypeptide

Selenoprotein

Sorbin and $\mathrm{SH} 3$ domain containing 1

Tropomyosin 3,gamma

Adenomatosis polyposis coli

A disintegrin and metallopeptidase domain 2

Anaplastic lymphoma kinase (Ki-1)

Interferon regulatory factor 4

Insulin-like growth factor 1 receptor

V-abl Abelson murine leukemia viral oncogene homologue 1

$\mathrm{V}$-abl Abelson murine leukemia viral oncogene homologue 2 (arg, Abelson-related gene)

Rho GTPase activating protein 5

Casein kinase 1, alpha 1

Casein kinase 2, alpha prime polypeptide

Immunity-related GTPase family $\mathrm{M}$ member 1

Integrin, alpha $\vee$ (vitronectin receptor, alpha polypeptide, antigen CD51)

p21 protein (Cdc42/Rac)-activated kinase 4

Abl interactor 2

Monoacylglycerol O-acyltransferase 1

A kinase (PRKA) anchor protein 11

Rho GTPase activating protein 26

AT rich interactive domain 4A (RBP1-like)

Coiled-coil domain containing 34

Cell division cycle 42 (GTP binding protein, $25 \mathrm{kDa}$ )

Cadherin 12, type 2 ( $\mathrm{N}$-cadherin 2)

CKLF-like MARVEL transmembrane domain containing 2B

Defensin beta 20

Eukaryotic translation initiation factor3, subunit $\mathrm{E}$

FERM domain containing 6

Ras-GTPase activating protein SH3 domain-binding protein 2

$\mathrm{G}$ protein-coupled receptor 143

4-hydroxyphenylpyruvate dioxygenase-like

Hydroxysteroid (17-beta) dehydrogenase 11

Platelet-derived growth factor receptor-like

Protein phosphatase 3, catalytic subunit, gamma isoform

Ring finger protein 139

Ribosomal protein $\mathrm{L} 7$

STE20-related kinase adaptor beta

Transmembrane 4 superfamily member 5
GenBank accession No.

NM_019765

NM_009609

NM_001024458

NM_011785

NM_010043

NM_177290

NM_008839

NM_009041

NM_053102

NM_001034962

NM_022314

NM_011701

NM_007462

NM_009618

NM_007439

NM_013674

NM_010513

NM_009594

NM_001136104

NM_009706

NM_146087

NM_009974

NM_008326

NM_008402

NM_001077403

NM_027470

NM_001198570

NM_026713

NM_001164503

NM_175164

NM_001081195

NM_026613

NM_009861

NM_001008420

NM_028524

NM_176950

NM_008388

NM_028127

NM_001080794

NM_010951

NM_146256

NM_053262

NM_026840

NM_008915

NM_175226

NM_011291

NM_172656

NM_178046

NM_029360

shRNAs were categorized by biological function using DAVID (database for annotation, visualization and integrated discovery).

migration promoters (csnk2a2, arid4a, irf4 and alk) and the inhibitors (mtmr1, dock3, myo5a and ptpn14) mediate their effects via the PI3K/AKT pathway. Furthermore, at the concentration used in the current study, the PI3K and AKT inhibitors effectively inhibited downstream signalling pathways and were without effects on cell viability (Supplementary Fig. 6). To gain a better understanding of the role of PI3K/AKT signalling in cell migration following specific gene knockdown, foxo1 and p70s6k1 (downstream components of the AKT pathway) were investigated. The phosphorylation of FOXO1 and p70S6K1 was increased after knockdown of the migration-inhibiting genes dock3, mtmr1, ptpn14 and myo5a, and after the upregulation of the migration-promoting genes (alk and irf4) (Supplementary Fig. 7). These results support that activation of the PI3K/AKT 


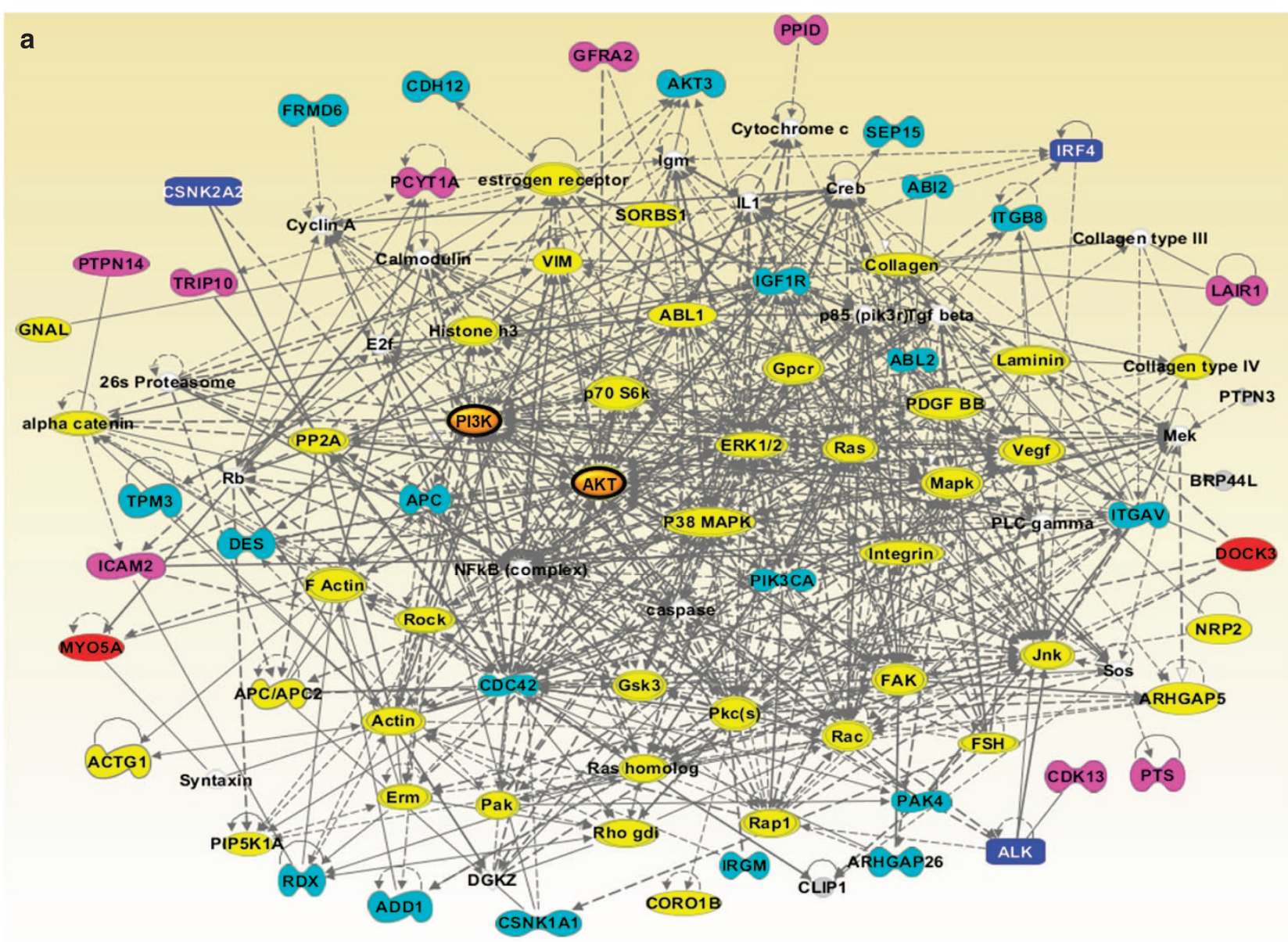

b

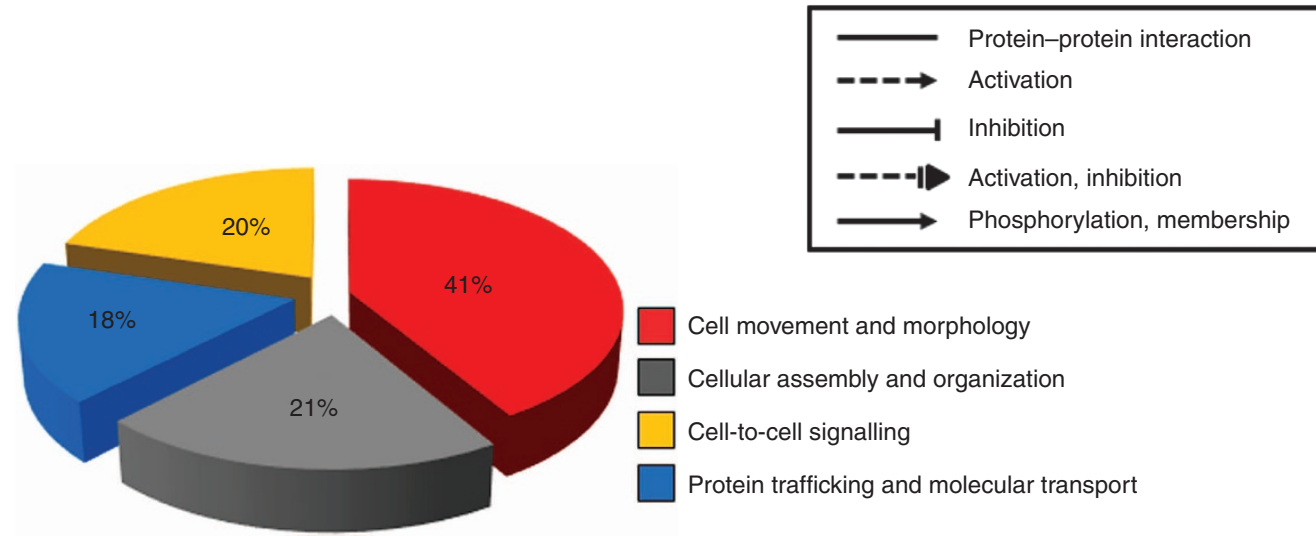

Figure 2 | Construction of the signalling network of cell migration regulators and their classification based on biological functions. (a) A relevant signalling network was constructed from the 91 cell migration-regulating genes identified by ingenuity pathway analysis (IPA). Newly identified cell migration regulators were linked to various previously reported cell movement signalling components (yellow). Green, cell migration-accelerating genes; blue, cell migration-accelerating genes validated by siRNA; pink, cell migration-impairing genes; red, cell migration-impairing genes validated by siRNA study. PI3K and AKT are highlighted in brown. (b) The biological functions of cell migration regulators were categorized by IPA analysis, which showed 41\% were cell movement and morphology related, $21 \%$ were cellular assembly and organization related, $20 \%$ were cell-to-cell signalling related and $18 \%$ were protein trafficking and molecular transport related.

pathway and downstream events, such as FOXO1 and p70S6K1 phosphorylation, appear to be critically required for diverse cell migration regulators identified by unbiased functional selection.

Unique role of $\mathbf{p 5 5} \gamma$ regulatory subunit of PI3K. In the next set of experiments, the cell migration-promoting gene alk was subjected to further investigation. Many receptor tyrosine kinases transduce their signals via specific interactions with $\mathrm{SH} 2$ domaincontaining proteins such as regulatory subunits of $\mathrm{PI} 3 \mathrm{~K}^{20}$. Therefore, we asked whether the receptor tyrosine kinase alk regulates the PI3K pathway through interaction with regulatory subunits of PI3K. Although anaplastic lymphoma kinase (ALK) physically interacted with both $\mathrm{p} 85 \alpha$ and $\mathrm{p} 55 \gamma$ regulatory subunits of PI3K (Fig. 8a,b), ALK overexpression enhanced phosphorylation of $\mathrm{p} 55 \gamma$, but not $\mathrm{p} 85 \alpha$, subunit (Fig. $8 \mathrm{c}$ ). The 


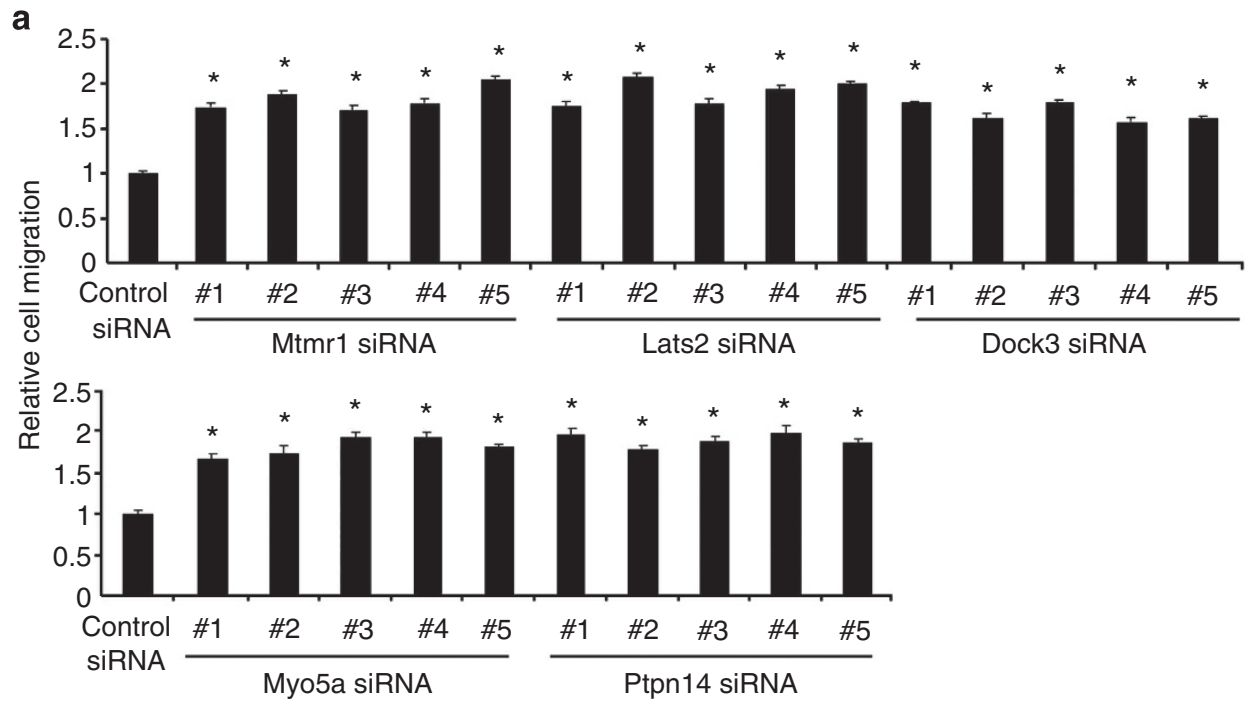

b
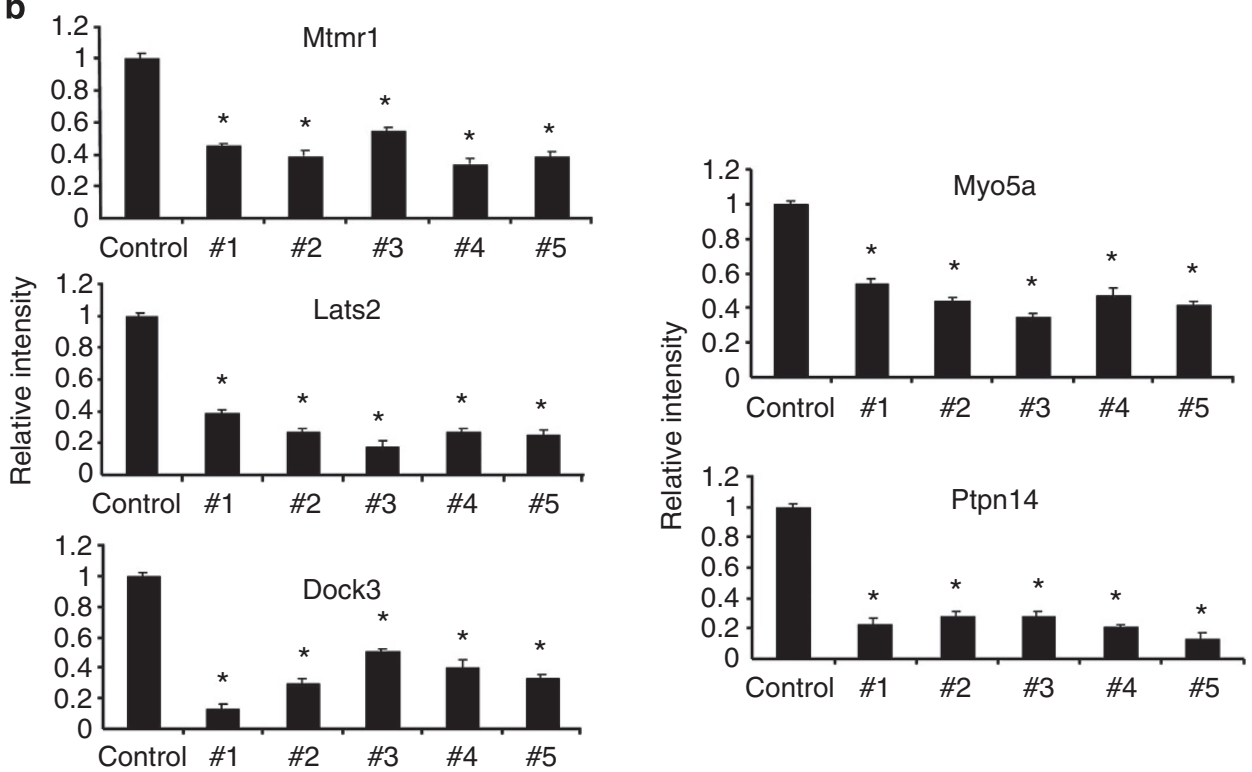

Figure 3 | Validation of the gene targets found by the RNAi-based functional selection. (a) NIH3T3 fibroblast cells were transiently transfected with control siRNA or one of five siRNAs (\#1 to \#5) targeting mtmr1, lats2, dock3, myo5a or ptpn14. At $24 \mathrm{~h}$ after transfection, wound-healing assays were performed to evaluate cell migration. (b) Efficiencies of siRNA-mediated target gene knockdown were confirmed by RT-PCR followed by densitometric analysis. (c) NIH3T3 cells were transiently transfected with control siRNA or one of five siRNAs (\#1 to \#5) for each target gene identified (csnk2a2, arid4a, ppp3cc, irf4 and alk). After $24 \mathrm{~h}$, wound-healing assays were performed to evaluate cell migration. Cell migration was quantified by measuring degrees of wound closure, as described in Methods. The results shown are means \pm s.d. $(n=3)$. ${ }^{\star} P<0.05$ represents significantly different from control siRNAtransfected cells. (d) Efficiencies of siRNA-mediated target gene knockdown were confirmed by RT-PCR and densitometric analysis. $\beta$-Actin was used as the internal control. The results are mean \pm s.d. $(n=3)$; ${ }^{\star} P$-values of $<0.05$ indicate significantly different from control siRNA-transfected cells.

ALK-induced phosphorylation of $\mathrm{p} 55 \gamma$ regulatory subunit of PI3K was accompanied by AKT phosphorylation (Fig. 8c) and translocation to the plasma membrane (Fig. 8d). GFP-fused AKT-PH domain was used to demonstrate the membrane translocation of $\mathrm{AKT}^{21}$. The critical role of $\mathrm{p} 55 \gamma$ and its phosphorylation in AKT activation and subsequent cell migration was further evaluated by siRNA-mediated knockdown of $p 55 \gamma$ (Fig. 8e,f and Supplementary Fig. 8) ${ }^{22}$. $p 55 \gamma$ siRNAs decreased ALK-induced AKT phosphorylation and cell migration, further supporting the unique role of p55 $\gamma$ subunit of PI3K in the Alk-promoted cell migration. These results indicate that ALK promotes cell migration by specifically interacting with p55 $\gamma$ subunit of PI3K, rather than more common p85 subunit (Supplementary Fig. 9a).

\section{Discussion}

We utilized lentivirus-based shRNA libraries targeting the entire mouse genome to enable genome-wide loss-of-function analysis by stable gene knockdown. Broad application of this shRNA library has already been reported in numerous studies on the identification of human disease-related genes ${ }^{23-28}$ and genes associated with other phenotypes of interest ${ }^{29-33}$. In the current study, we aimed to identify diverse cellular pathways whose knockdowns have positive or negative effects on cell migration phenotypes. Some of the cell migration-regulating genes identified in the present study have well-established links to cellular motility, which validates the selection approach used. We also identified a number of previously uncharacterized cell migration regulators not previously identified by RNAi 


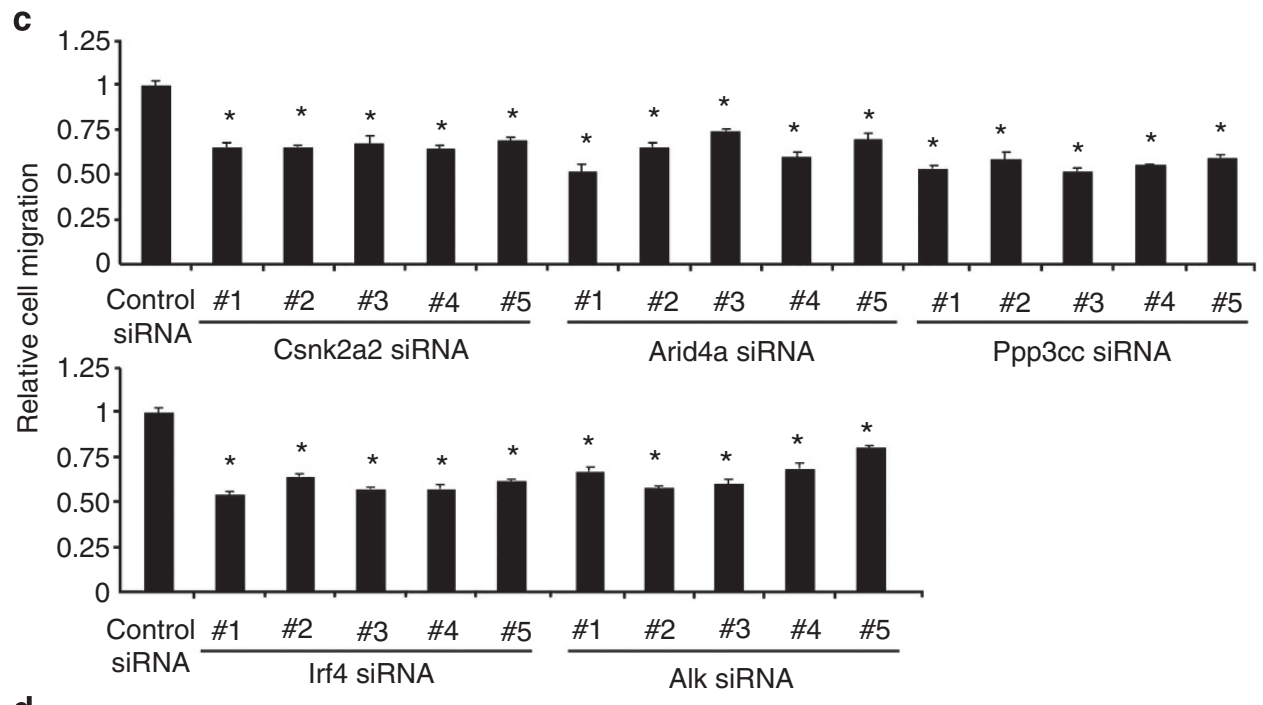

d
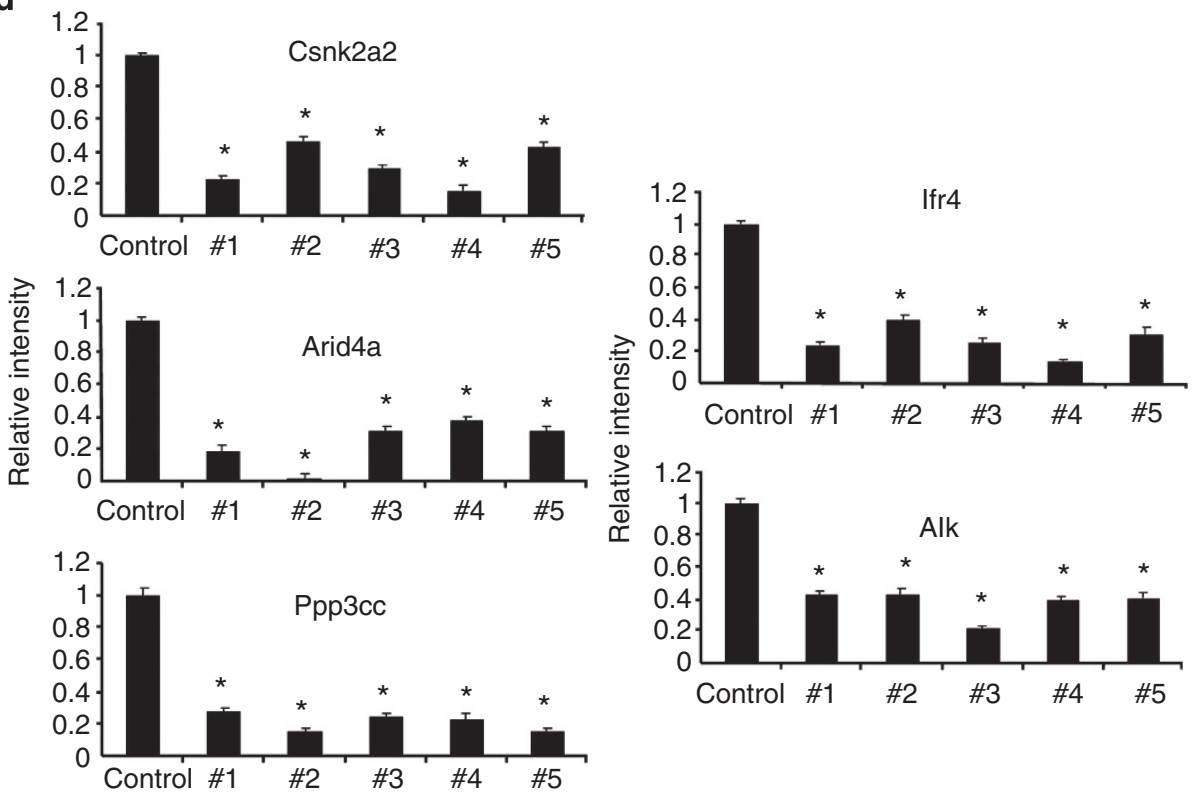

Figure 3 | Continued.

screening. Importantly, several novel cell migration regulators that were investigated in more detail were found to show marked downstream signalling convergence on the PI3K/PTEN/AKT pathway, which emphasizes the central roles of this pathway in cell migration.

Although the RNAi-based functional screening of cell migration regulators has been previously conducted, our approach differs in several ways. First, we performed unbiased genomescale shRNA selection, rather than screening, as phenotypedriven selection processes reduce false positives and as cells with given phenotypes are separated and enriched several times. The selection method used in the present study is straightforward and cost-effective as compared with array-based RNAi screen. Second, we used cells of fibroblast origin, which have higher baseline motilities than epithelial cells, as this allowed us to select cell migration accelerators and impairers at the same time. In the event, the selection method used identified many migrationpromoting and -inhibiting genes not previously reported. Third, we used direct cloning and sequencing of half-hairpin barcodes, rather than microarray analysis, to identify selected shRNAs. This process enabled the unbiased and direct selection of genes contributing to a given phenotype, as microarray-based identification involves statistical evaluation and a degree of ambiguity. In this method, however, it was difficult to assess how representative the selected shRNAs are to the starting shRNA library.

Among the functionally diverse 91 hits identified in the primary screen, targets of 13 shRNAs were protein kinases and protein phosphatases. Our results support the validity of the selection methods used and emphasize the critical roles played by protein kinases and phosphatases in cell motility regulation. Some of these kinases and phosphatases were included in our initial validation list, which comprised five migration-accelerating shRNA candidates (mtmr1, lats2, dock3, myo5a and ptpn14)

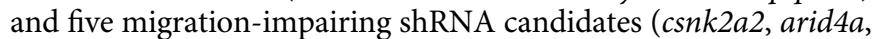
$p p p 3 c c$, irf4 and alk). In later studies, we found that the actions of some of these cell migration regulators, namely, $m t m r 1$, dock3, myo5a, ptpn14, csnk2a2, alk, irf4 and arid4a (but not lats2 or ppp3cc), are strongly dependent on the PI3K/PTEN/AKT pathway (Supplementary Fig. 9b). Additional validation studies of 36 hits showed $71.7 \%$ overall correlation between the results of primary screen and secondary test. 
a
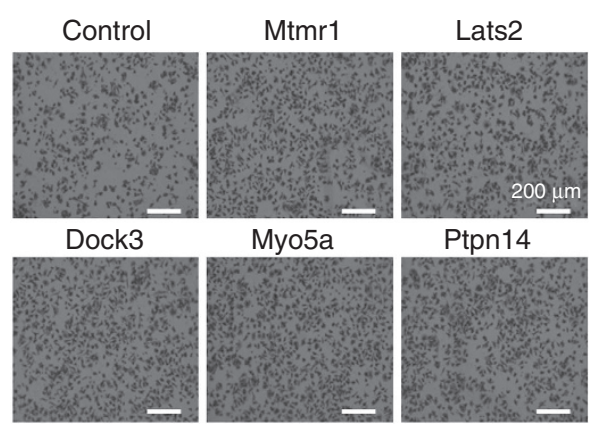

Ptpn14

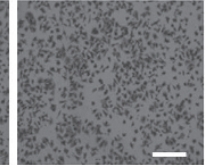

b
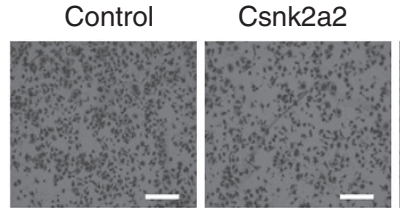

Ppp3cc

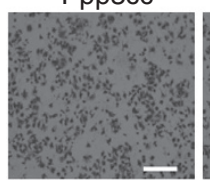

Irf4

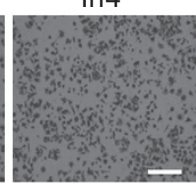

Arid4a

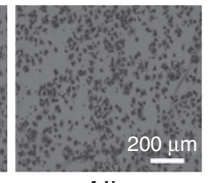

Alk

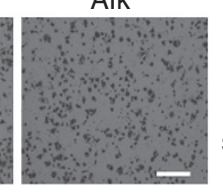

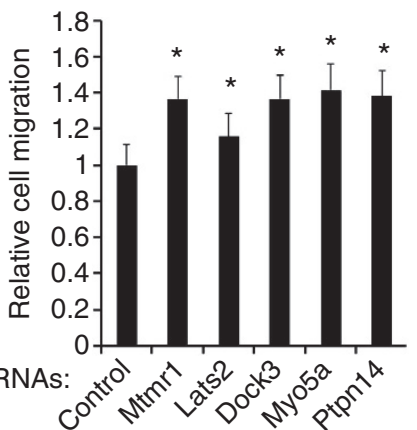

C
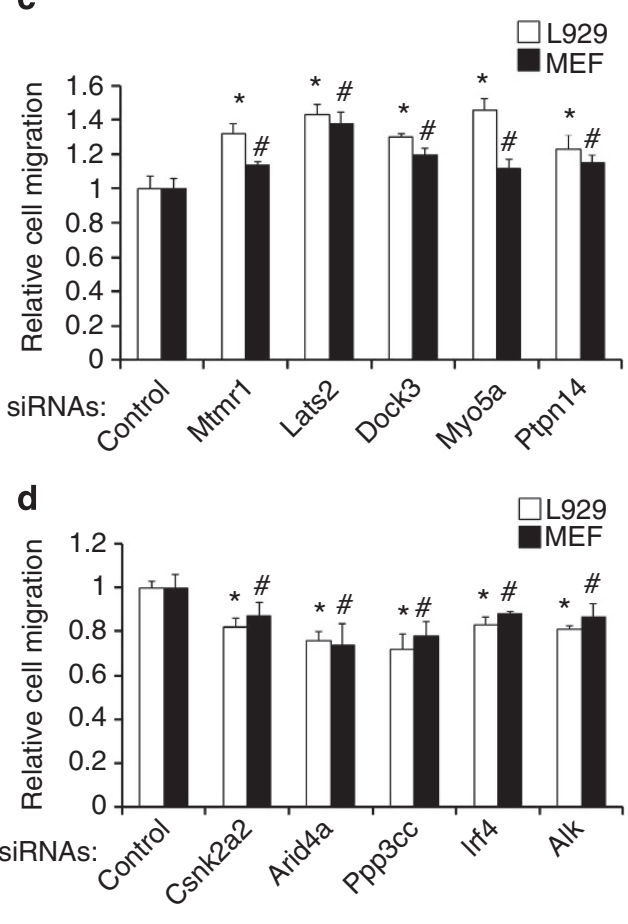

Figure 4 | Validation of shRNA hits by three-dimensional cell migration assay. (a,b) NIH3T3 fibroblast cells were transiently transfected with siRNAs targeting cell migration inhibitors (a) or promoters (b). One siRNA was used for each target: mtmr1 (\#5), lats2 (\#3), dock3 (\#1), myo5a (\#3), ptpn14 (\#5), csnk2a2 (\#1), arid4a (\#1), ppp3cc (\#3), irf4 (\#4) or alk (\#3). \#1 to \#5 indicate the siRNAs used for validation in Fig. 3. After $24 \mathrm{~h}$ of transfection, NIH3T3 fibroblast cells $\left(4 \times 10^{4}\right.$ cells/well) were seeded onto transwell inserts and incubated at $37^{\circ} \mathrm{C}$ for $6 \mathrm{~h}$ (a) cell migration-accelerating siRNAs) or $9 \mathrm{~h}$ (b; cell migration-impairing siRNAs). Non-migrated cells were removed from the upper face of the transwell insert using a cotton swab. Cells that migrated through membranes were stained and counted in five randomly selected fields. The results are representative of three independent experiments (left) or mean \pm s.d. $\left(n=3\right.$ ) (right). ${ }^{\star} P<0.05$ represents significantly different from control siRNA-transfected NIH3T3 cells. Scale bar, $200 \mu$ m. (c,d) L929 fibroblast cells or mouse embryonic fibroblasts (MEFs) were transiently transfected with cell migration-accelerating (c) or impairing (d) siRNAs identified from the screen. After $24 \mathrm{~h}$ of transfection, L929 or MEF cells $\left(4 \times 10^{4}\right.$ cells/well) were seeded onto the transwell culture inserts and incubated at $37{ }^{\circ} \mathrm{C}$ for 6-9 h. After incubation, non-migrated cells were removed from the upper face of the transwell culture insert using a cotton swab. The cells that migrated across the membrane were stained and counted as described in the main text. The results are mean \pm s.d. $(n=3)$. ${ }^{\star} P<0.05$ represents significantly different from control siRNA-transfected $L 929$ cells. ${ }^{\#} P<0.05$ represents different from control siRNA-transfected MEF cells.

Several of the cell migration-regulating genes identified in this study have been previously associated with the PI3K/PTEN/AKT pathway. Casein kinase 2 (CK2 or $\operatorname{csnk} 2)$ is a physiologically relevant PTEN kinase, and CK2-mediated phosphorylation inhibits PTEN function, which in turn increases PIP3 levels, and thus AKT phosphorylation. The expression levels and activities of CK2 subunits have been reported to be increased three- to fivefold in many human cancer and tumour cell lines ${ }^{34-36}$. Protein tyrosine phosphatase non-receptor type 14 (ptpn14) is frequently mutated in a variety of human cancers. However, the cell signalling pathways regulated by Ptpn14 remain largely unknown ${ }^{37}$. In the present study, protein-protein interaction between PTPN14 and PTEN was identified by IPA analysis (Supplementary Fig. 2e), which suggests that PTPN14 regulates cell migration by modulating PTEN activity. The physiological role of myotubularin-related protein 1 ( $m$ tmr 1 ) has not been clearly determined, although recent studies have shown that $m t m r l$ is a phosphatase, like myotubularin, and can dephosphorylate both PtdIns3P and PtdIns3,5P2 in vitro ${ }^{38,39}$, which suggests its possible involvement in the PI3K/PTEN/AKT pathway.

Some of the cell migration regulators identified in this study appear to act independently of the PI3K/PTEN/AKT pathway. $P p p 3 c c$, also known as calcineurin A subunit, is a $\mathrm{Ca} 2+-$ and calmodulin-dependent serine/threonine protein phosphatase. In a recent study, calcineurin was shown to promote tumour cell invasion via transcriptional factor NFAT in breast cancer metastasis $^{40}$. Lats2 is a serine/threonine protein kinase belonging to the tumour suppressor family, and has been reported to participate in p53 signalling ${ }^{41}$ and to be implicated in cell cycle regulation, apoptosis, centrosome duplication and genomic stability ${ }^{42-44}$. Furthermore, our findings suggest that both $p p p 3 c c$ and lats 2 promote or inhibit cell migration, respectively, via a mechanism not involving the PI3K/PTEN/ AKT pathway.

In the present study, the activations of FOXO1 and p70S6K1 were investigated as signalling events downstream of the PI3K/ PTEN/AKT pathway to determine the mechanistic basis of impaired or accelerated cell migration following specific gene knockdown. Forkhead box O (FOXO) proteins, which include FOXO1, FOXO3, FOXO4 and FOXO6, regulate many cancerrelated phenotypes by inhibiting nuclear transcription factors. In the previous studies, activation of AKT led to FOXO1 phosphorylation and nuclear exclusion ${ }^{45}$. Furthermore, the nuclear exclusion of FOXO1 abolishes its inhibitory effect on Runx2 activity, and therefore enhances Runx2-mediated gene transcription and the subsequent migration/invasion of cancer cells ${ }^{46}$. In addition, the AKT-mediated phosphorylation of FOXO1 induces its degradation by proteasome ${ }^{47,48}$. Consistent 
a
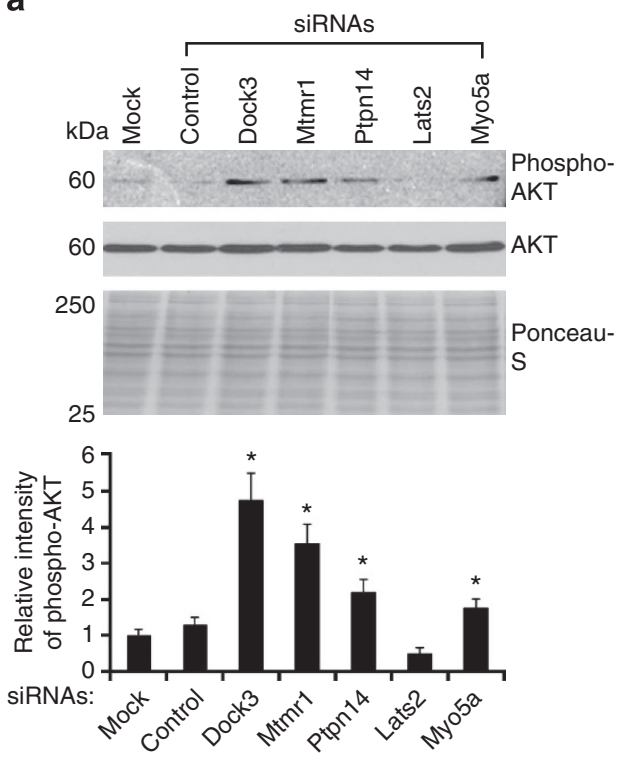

d

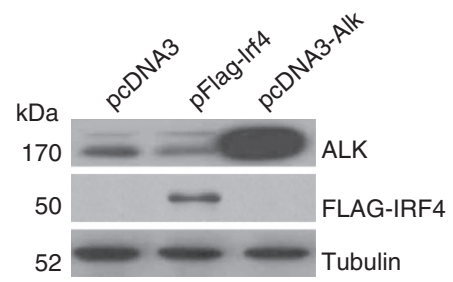

b

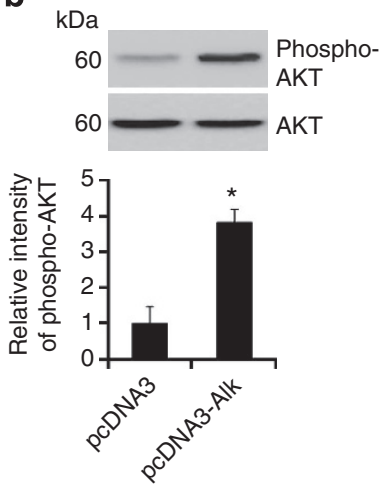

C
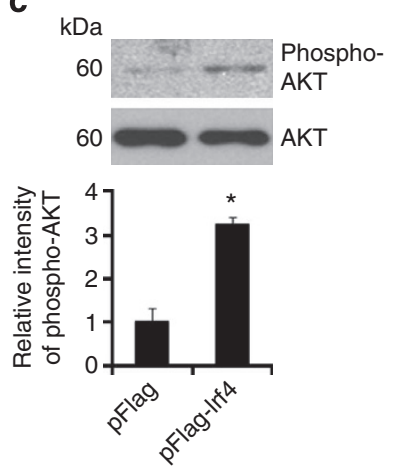

Figure $\mathbf{5}$ | Induction of AKT activation by the selected cell migration regulators. NIH3T3 fibroblast cells were transiently transfected with control siRNA, siRNAs against dock3 (\#1), mtmr1 (\#5), ptpn14 (\#5), lats2 (\#3) or myo5a (\#3) (a) or empty vectors (pcDNA3 or pFlag), alk or irf4 expression constructs (pcDNA3-alk or pFlag-irf4) (b,c). After $48 \mathrm{~h}$, levels of phosphorylated AKT (phospho-AKT at Ser473) or total AKT protein were evaluated by western blot analysis (upper). Ponceau $S$ staining was performed to confirm equal sample loading. The results of densitometric analysis are also shown (lower). The results shown are means \pm s.d. $(n=3)$; ${ }^{\star} P$-values of $<0.05$ indicate significantly different from control siRNA- or empty vector-transfected cells. (d) Overexpression of ALK or IRF4 in transfectants was confirmed by western blotting using an anti-ALK or -FLAG-tag antibody. Tubulin was used as the internal control.

with previous findings, we found that the level of FOXO1 was reduced after phosphorylation (Supplementary Fig. 7). In a previous study, $\mathrm{mTORC} 1$ and $\mathrm{mTORC} 2$ signalling pathways were found to be involved in cytoskeletal rearrangement and cell migration ${ }^{49,50}$. In particular, the activations of mTORC1 and p70S6K1 were found to be essential for the migration and invasion of cancer and non-cancer cells via cytoskeletal rearrangement ${ }^{49,51}$. In the present study, the phosphorylation of AKT, FOXO1 and p70S6K1 was found to be associated with increased cell migration, which supports the notion that FOXO1 and p70S6K1 are downstream signalling components of PI3K/ PTEN/AKT.

The PI3K family is categorized into three classes (class I, II, and III) and various subclasses based on their structure, substrate specificity and regulation. The class I PI3K is the bestcharacterized subfamily present in all cell types. They consist of a p110 catalytic subunit $(\alpha, \beta, \gamma$ or $\delta)$ and a regulatory subunit $(\mathrm{p} 85 \alpha, \mathrm{p} 85 \beta, \mathrm{p} 55 \alpha, \mathrm{p} 55 \gamma \text { or } \mathrm{p} 50 \alpha)^{52}$. Regulatory subunits are required to recruit the p110 catalytic subunit to specific cellular locations, thereby regulating its catalytic activity. The different regulatory subunits associate with distinct receptor tyrosine kinases ${ }^{53}$ and show the specificity in mediating different PI3K signalling pathways ${ }^{54}$. However, the mechanisms by which these regulatory subunits regulate specific signalling pathways are not fully understood. p55 $\gamma$, also known as p55PIK, binds the catalytic subunits and modulates PI3K activity in a manner similar to p $85 \alpha$ and $p 85 \beta^{54,55}$. Our data indicate that $\mathrm{p} 55 \gamma$ was critically involved in the receptor tyrosine kinase Alk-promoted PI3K/AKT activation and cell migration. In the previous reports, p55 regulatory subunit of PI3K promoted cell cycle progression ${ }^{56}$, DNA synthesis ${ }^{57}$, proliferation and differentiation of leukemia cells $^{58}$, and tumour angiogenesis via regulation of NF- $\mathrm{KB}$ signalling or expression of VEGF-A ${ }^{59}$. Furthermore, the protein levels of p55 $\gamma$ increased in colorectal, gastric and ovarian cancers $56,59,60$. PI $3 \mathrm{~K}$ also plays an important role in the multiple steps of neurite outgrowth during nerve growth factorstimulated differentiation and in the brain development ${ }^{61}$.

ALK was originally identified as an oncogene in human anaplastic large cell lymphoma and neuroblastoma ${ }^{62}$. ALK displays the classical structural features of a receptor tyrosine kinase. In the previous studies, ALK mediated several signaltransduction pathways, including JAK/STAT, RAS/MAPK, PI3K and PLC $\gamma$, and modulated various cellular functions, such as proliferation, angiogenesis, metabolism and migration ${ }^{63,64}$. An important role of ALK in the development and function of the nervous system has also been reported ${ }^{65}$. Brain expression of ALK

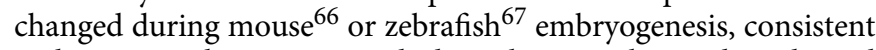
with our results. However, little is known about physiological functions of ALK and its cognate ligands in vertebrate. In this study, for the first time, we have identified the $\mathrm{p} 55 \gamma$ regulatory subunit of PI3K as a target molecule of ALK during mouse fibroblast cell migration (Supplementary Fig. 9a). Physical 

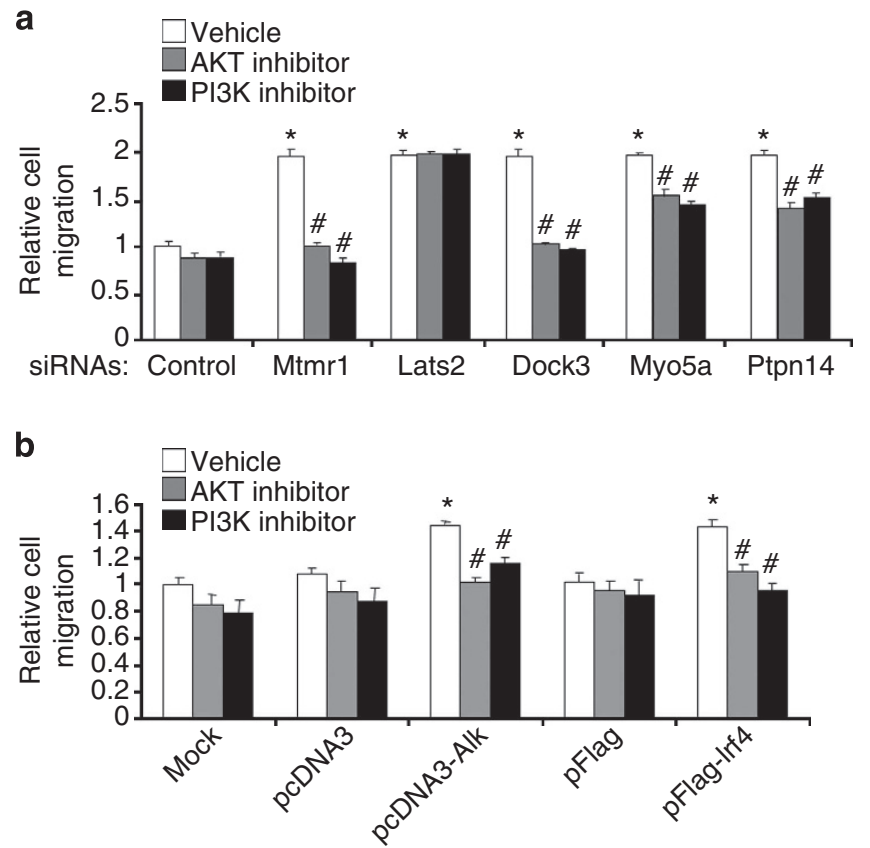

Figure 6 | Dependences of selected cell migration regulators on the PI3K/AKT pathway. (a) NIH3T3 cells were transiently transfected with control siRNA or siRNAs targeting $m t m r 1$ (\#5), lats2 (\#3), dock3 (\#1), myo5a (\#3) or ptpn14 (\#5). After $24 \mathrm{~h}$, wound-healing assay was conducted and cell migration was quantified in the presence or absence of AKT inhibitor $(5 \mu \mathrm{M})$ or PI3K inhibitor (LY294002; $15 \mu \mathrm{M})$. The results shown are means \pm s.d. $(n=3)$. ${ }^{*} P$-values of $<0.05$ indicate significantly different from control siRNA-transfected cells; ${ }^{*} P$-values of $<0.05$ indicate significantly different from the corresponding siRNA-transfected cells not treated with inhibitor. (b) Alternatively, NIH3T3 cells were transiently transfected with empty vector ( $p c D N A 3$ ) or alk or irf4 expression constructs ( $p c D N A 3$-alk or pFlag-irf4) for $24 \mathrm{~h}$. Wound-healing assay was performed to evaluate cell migration in the presence or absence of AKT inhibitor $(5 \mu \mathrm{M})$ or PI3K inhibitor (LY294002; $15 \mu \mathrm{M})$. The results shown are mean \pm s.d. $(n=3)$. ${ }^{\star} P$-values of $<0.05$ indicate significantly different from $p c D N A 3$ or pFlag empty vector transfected cells; ${ }^{*} P$-values of $<0.05$ indicate significantly different from the cells under a similar condition without the inhibitor treatment.

interaction between ALK and p55 $\gamma$, ALK-induced phosphorylation of $\mathrm{p} 55 \gamma$, and $\mathrm{p} 55 \gamma$ knockdown studies supported these findings. NIH3T3 cells express three isoforms of AKT. When the involvement of AKT isoforms in the ALK-induced cell migration was examined by knockdown of individual isoforms of akt (akt1, akt2 and akt3), all three isoforms of AKT were found to play an important role in the ALK-induced migration (Supplementary Fig. 10). Although the critical role of $\mathrm{p} 55 \gamma$ in the ALK/AKTinduced cell migration has been demonstrated in the current study, it does not necessarily indicate that all the migrationrelated signalling is mediated via ALK/AKT and $\mathrm{p} 55 \gamma$. Also, the phosphorylation of the regulatory subunit $\mathrm{p} 55 \gamma$ should not be regarded as a definitive indication for increased migration, as the cell migration depends on the relevant downstream PI3K targets and additional regulators. Further studies need to be conducted to better assess the importance of p55 $\gamma$ in cell migration in general.

In summary, the selection method used in the present study allowed us to identify a large number of genes that modulated cell migration. Furthermore, many of the cell migration-regulating genes identified have not been previously associated with cell migration. Although the precise regulatory mechanisms responsible for the effects of these cell migration regulators will only be

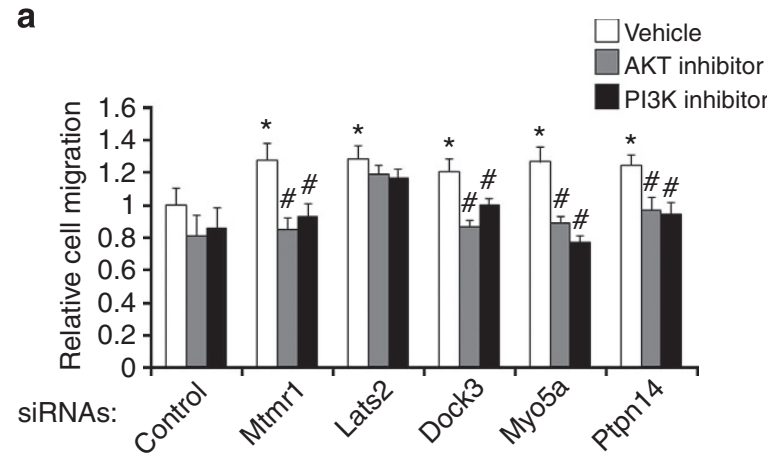

b

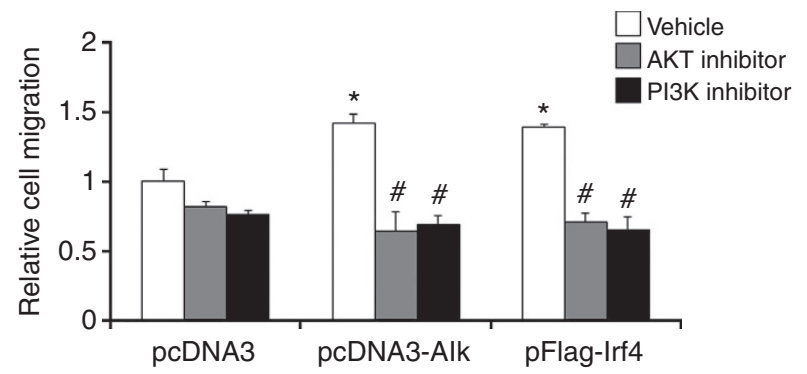

Figure 7 | Dependence of selected cell migration regulators on the PI3K/ AKT pathway determined by three-dimensional cell migration assay. NIH3T3 cells were transiently transfected with either siRNAs targeted for mtmr1, lats2, dock3, myo5a and ptpn14 (a) or alk and irf4 expression constructs ( $p c D N A 3$-alk or pFlag-irf4) (b). After $24 \mathrm{~h}$ of transfection, $\mathrm{NIH} 3 \mathrm{~T} 3$ fibroblast cells $\left(4 \times 10^{4}\right.$ cells/well) were seeded onto the transwell culture inserts and incubated in the presence or absence of either AKT inhibitor $(5 \mu \mathrm{M})$ or PI3K inhibitor (LY294002; $15 \mu \mathrm{M})$ at $37^{\circ} \mathrm{C}$ for $6 \mathrm{~h}$. After incubation, non-migrated cells were removed from the upper face of the transwell culture insert. The cells that migrated across the membrane were stained and counted as described in the main text. The results are mean \pm s.d. $(n=3)$. ${ }^{\star} P<0.05$ represents significantly different from control siRNA-transfected cells; ${ }^{\#} P<0.05$ represents significantly different from the corresponding siRNA-transfected cells without the inhibitor treatment.

determined by additional studies, the candidate gene list obtained by the current selection process provides an integrated view regarding cell migration.

\section{Methods}

Reagents and cells. Specific small-molecule inhibitors, such as Akt inhibitor (1L6-hydroxymethyl-chiro-inositol-2-(R)-2-O-methyl-3-O-octadecyl-sn-glycerocarbon) and PI3K inhibitor (LY294002), were purchased from Calbiochem (La Jolla, CA). Puromycin and mitomycin C were purchased from Sigma-Aldrich (St Louis, MO). All other chemicals, unless otherwise stated, were obtained from Sigma-Aldrich. NIH3T3 mouse fibroblast cells, L929 mouse fibroblast cells and HEK293T human embryonic kidney epithelial cells were maintained in Dulbecco's modified Eagle's media (DMEM) supplemented with $10 \%$ heat-inactivated fetal bovine serum (FBS) (Invitrogen, Carlsbad, CA), $100 \mathrm{U} \mathrm{ml}^{-1}$ penicillin and $100 \mu \mathrm{g} \mathrm{ml}^{-1}$ streptomycin (Gibco-BRL, Rockville, MD). Phoenix Ampho cells were cultured in DMEM containing 10\% FBS (Lonza, Walkersville, MD), hygromycin $\mathrm{B}\left(300 \mu \mathrm{g} \mathrm{ml}^{-1}\right)$ and diphtheria toxin $\left(1 \mu \mathrm{g} \mathrm{ml}{ }^{-1}\right)$ at $37^{\circ} \mathrm{C}$ and $5 \% \mathrm{CO}_{2}$. MEF cell cultures were prepared from 13.5-day-old embryos of Institute for Cancer Research (ICR) mice ${ }^{68}$.

Lentivirus production and titration. A 63,996 pooled lentiviral shRNA library targeting 21,332 mouse genes was generated by the transient transfection of HEK293T cells with $p H A G E$-mir30-RFP-shRNA (targeting the mouse genome), $p V S V-G, p T a t, p P M 2$ and $p R e v$ using LipofectAMINE 2000 (Invitrogen), in accordance with the manufacturer's instructions. The mir30-based shRNA constructs have been previously described ${ }^{14}$. Cell-free supernatants were collected 2-3 days after transfection and were subsequently used to transduce NIH3T3 cells in the presence of $8 \mu \mathrm{g} \mathrm{ml}^{-1}$ polybrene. After $24 \mathrm{~h}$, virus-containing supernatants 
were removed by centrifugation. To optimize conditions for viral infection and to monitor virus titres, NIH3T3 fibroblast cells $\left(1 \times 10^{6}\right.$ cells $)$ were seeded onto 100$\mathrm{mm}$ culture plates $18 \mathrm{~h}$ before infection and then incubated with $1 \mathrm{ml}$ of red fluorescence protein-carrying lentiviral stock for $6-8 \mathrm{~h}$, in the presence of polybrene $\left(8 \mu \mathrm{g} \mathrm{ml}^{-1}\right)$. Fresh DMEM/10\% FBS (2 ml) containing polybrene $\left(8 \mu \mathrm{g} \mathrm{ml}^{-1}\right)$ was then added to the culture and incubation was continued. After another $24 \mathrm{~h}$, cells were removed from plates, and infection efficiencies and viral titres were determined by observing red fluorescence protein under a fluorescence a

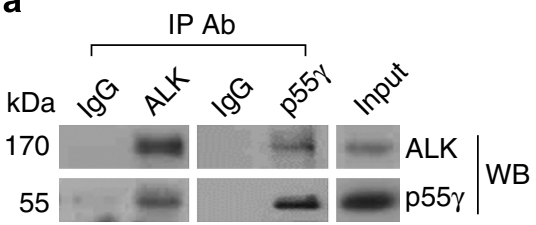

b

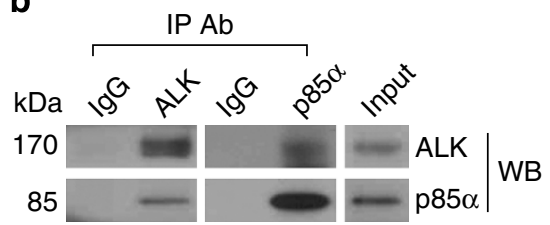

C

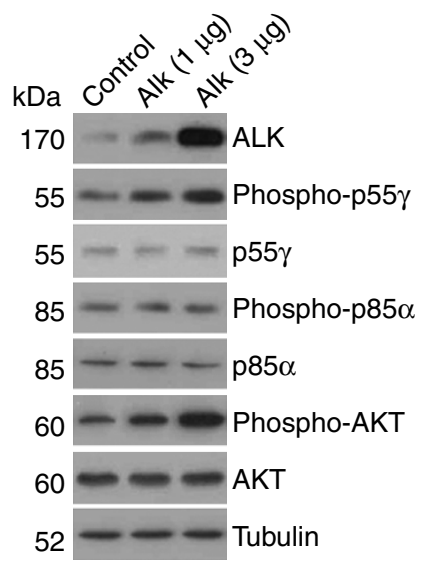

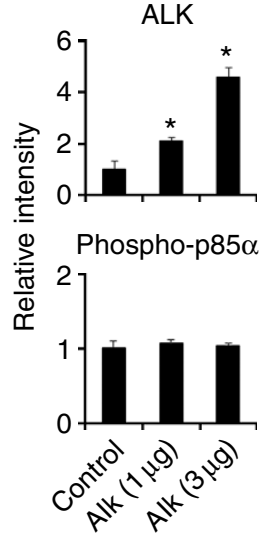

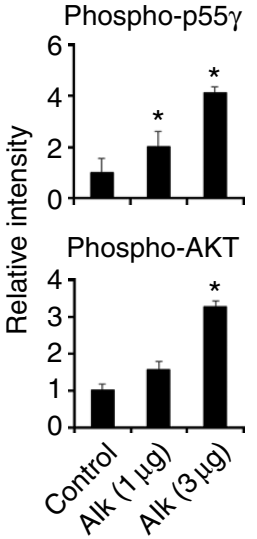

d

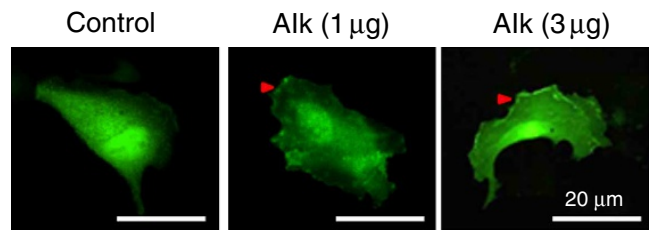

e
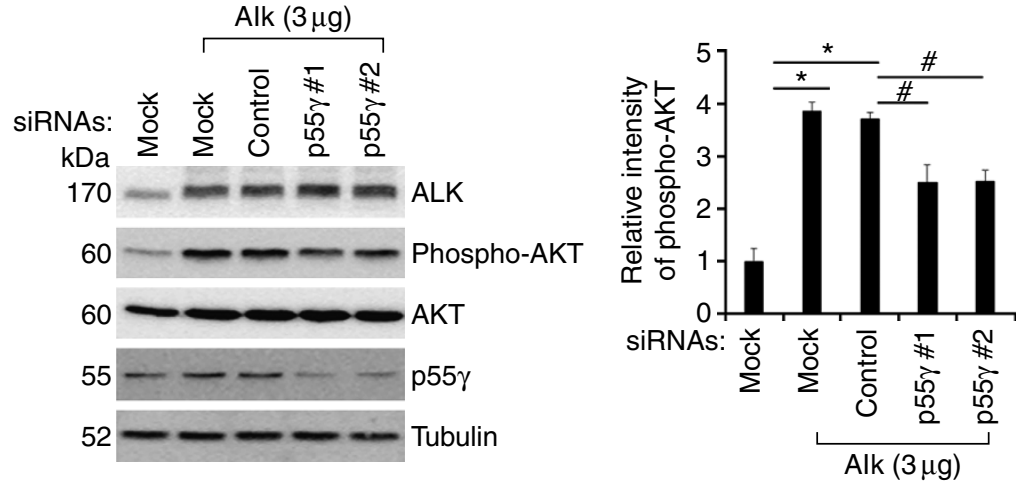

$\mathbf{f}$

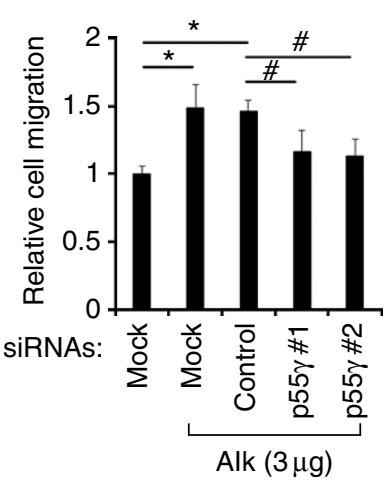


microscope (Olympus BX50; Tokyo). The titre of the viral shRNA library was $\sim 7.23 \times 10^{6}$ p.f.u. $\mathrm{ml}^{-1}$

Selection of cell migration regulators using shRNA library. NIH3T3 fibroblast cells were seeded at the density of $1 \times 10^{6}$ cells $/ 100-\mathrm{mm}$ culture plate. Three plates of NIH3T3 cells were infected with the lentiviral shRNA library at an m.o.i. of 1 in the presence of polybrene $\left(8 \mu \mathrm{g} \mathrm{ml}^{-1}\right)(45 \times$ representation for each shRNA). Two days after infection, cells were puromycin $\left(10 \mathrm{ng} \mathrm{ml}^{-1}\right)$-selected for 7 days, detached, seeded onto transwell culture inserts $(8-\mu \mathrm{m}$ pore membrane; Millipore, Billerica, MA) in 24-well plates $\left(\sim 4 \times 10^{4}\right.$ cells/transwell culture insert/well $)$ and incubated at $37^{\circ} \mathrm{C}$ for either 5 or $24 \mathrm{~h}$. The 5 -h time point was used to select migration enhancers and $24 \mathrm{~h}$ was used to identify inhibitors of migration. Upper and lower transwell chambers were filled with media containing $0 \%$ or $10 \%$ FBS, respectively, to induce the directional movement of cells. Infected cells $\left(\sim 3 \times 10^{6}\right.$ cells) were divided and placed onto 72 transwell culture inserts. Cells that did or did not migrate were collected by trypsinizing cells adherent to the lower or upper faces of transwell culture inserts, respectively, and then reseeded onto transwell culture inserts. Cells from 72 individual wells were then allowed to re-migrate five times for enrichment purposes. Selected cells from the 72 transwell culture inserts were then combined. To identity the shRNAs integrated into the combined cells, a small amount $(50 \mathrm{ng})$ of genomic DNA separately isolated from migrating or nonmigrating cells was subjected to PCR. shRNA segments were amplified using the primer set: forward primer JH353F, TAGTGAAGCCACAGATGTA; reverse primer $B C 1 R$, CCTCCCCTACCCGGTAGA ${ }^{14}$. The resulting PCR fragments ( $453 \mathrm{bp}$ ) were gel-purified, cloned and sequenced ${ }^{69}$ (Fig. 1). All clones were sequenced. The sequences of 22-27 nt, corresponding to the half-hairpins of shRNAs, were used to identify shRNAs.

Validation of primary hits using retroviral shRNAs. Retroviral shRNAs were generated by transient transfection of Phoenix Ampho packaging cells with each shRNA using Lipofectamine 2000 in accordance with the manufacturer's instructions. Cell-free supernatants were harvested 2-3 days after transfection and subsequently were used to transduce NIH3T3 fibroblast cells in the presence of $8 \mu \mathrm{g} \mathrm{ml}^{-1}$ polybrene. To optimize conditions of viral infection and to monitor virus titre, a test construct of the retroviral vector carrying GFP ( $p F B-h r G F P$ ) (Stratagene) was used. For infection of NIH3T3 fibroblast cells, cells $\left(1 \times 10^{5}\right.$ cells/ well) were seeded onto $60-\mathrm{mm}$ culture plates $18 \mathrm{~h}$ before infection and incubated with $1 \mathrm{ml}$ of virus stock for $6-8 \mathrm{~h}$ in the presence of polybrene $\left(8 \mu \mathrm{g} \mathrm{ml}^{-1}\right)$. Then, $2 \mathrm{ml}$ of fresh DMEM/10\% FBS containing polybrene $\left(8 \mu \mathrm{g} \mathrm{ml}{ }^{-1}\right)$ was added to the culture and the incubation was continued. After another $24 \mathrm{~h}$, cells were removed from the plates, and infection efficiency and viral titre were determined by the count of GFP-positive cells using FACS. NIH3T3 fibroblast cells were infected with the retroviral shRNA at an m.o.i. of 1-3. For high-throughput screening, Essen Incucyte and Woundmaker (Essen BioScience, Ann Arbor, MI) were used. In brief, the cell migration algorithm analyzed each image and assigned a well-specific wound mask that corresponded to the initial scratch wound. Scratch wound mask followed migrating cells as they moved into the initial wound, creating a measurement of relative wound density or wound closure.

Pathway and network analyses. The accession numbers of identified DNAs were exported to IPA (Ingenuity Systems) for network analysis. The statistical significance of each network or list was determined by IPA using Fisher's Exact test $(P<0.05)$. IPA was also used to construct networks of protein-protein and other regulatory interactions. For pathway construction, the IPA database used currently available knowledge on genes, proteins, chemicals, normal and disease cellular processes, signalling and cellular functions.

Transient transfection with siRNA or cDNA. Desalted and preannealed siRNA duplexes were purchased from Genolution Pharmaceuticals (Seoul, Korea). The
siRNAs were designed using a proprietary algorithm devised by Genolution Pharmaceuticals. Sequence information for the siRNAs used for validation is given in Supplementary Table 6. To knockdown specific gene expression, NIH3T3 mouse fibroblast cells were transfected with siRNAs at a final concentration of $10 \mathrm{nM}$ using LipofectAMINE (Invitrogen), according to the manufacturer's instructions. Cells were harvested $24-48 \mathrm{~h}$ after transfection, and the potencies of siRNAs to silence gene expression were measured by RT-PCR or western blot analysis. A control random sequence siRNA was also purchased from Genolution Pharmaceuticals and used as negative control. The full-length cDNAs of mouse alk or irf4 were cloned into the expression vectors $p c D N A 3$ or pFlag, respectively. NIH3T3 fibroblast cells in six-well plates were transiently transfected with $3 \mu \mathrm{g}$ of alk or irf4 cDNA using LipofectAMINE. The empty vector $p c D N A 3$ or $p F l a g$ was used as control for the transient expression of alk or irf4. One or two days after transfection, cells were used for experiments. The expressions of alk and irf4 mRNAs and proteins in transient transfectants were confirmed by RT-PCR or western blot analysis, respectively.

Reverse transcription-PCR. Total RNA was extracted from NIH3T3 cells in sixwell plates using TRIzol reagent (Invitrogen), according to the manufacturer's instructions. Reverse transcription was conducted using Superscript II (Invitrogen) and oligo(dT) primer. PCR amplification, using specific primer sets, was carried out at an annealing temperature of $55-60^{\circ} \mathrm{C}$ for $20-30$ cycles. PCR was performed by using a DNA Engine Tetrad Peltier Thermal Cycler (MJ Research, Waltham, MA). To analyse PCR products, $10 \mu \mathrm{l}$ of each PCR reaction product was electrophoresed on $1 \%$ agarose gel followed by ethidium bromide staining and detection under UV light. $\beta$-Actin was used as internal control. The nucleotide sequences of the primers used were based on published cDNA sequences (Supplementary Table 8).

Wound-healing assay. For the in vitro wound-healing assay, a scratch was created by using a $10-\mu$ l pipette tip on confluent cell monolayers in 24 -well culture plates, and then DMEM containing $10 \% \mathrm{FBS}, 100 \mathrm{U} \mathrm{ml}^{-1}$ penicillin and $100 \mu \mathrm{g} \mathrm{ml}^{-1}$ streptomycin was added. Cells were then incubated at $37^{\circ} \mathrm{C}$ under $5 \% \mathrm{CO}_{2}$ to enable migration into wounds, which were then observed under a light microscope (Olympus CK2; X100). To rule out confounding effect of cell proliferation, cells were treated with the mitosis inhibitor mitomycin- $\mathrm{C}$ at a final concentration of $10 \mu \mathrm{g} \mathrm{ml}^{-1}$ for $2 \mathrm{~h}$ before wounding. Relative cell migration distances were calculated by subtracting final wound widths from initial values ${ }^{70}$. Three nonoverlapping fields were selected and examined per well (three wells per experimental group). The results are presented as fold increases in migration distance versus control condition.

Three-dimensional cell migration assay. Cell migration was also measured using transwell culture inserts (8- $\mu \mathrm{m}$ pore membrane; Millipore), according to the manufacturer's instructions. In brief, cells were transfected with the siRNAs of migration accelerating or impairing genes. At $24 \mathrm{~h}$ after transfection, cells were harvested by trypsinization, resuspended in DMEM and added to upper wells at $4 \times 10^{4}$ cells/well. Growth media were placed into base wells, which were separated from top wells by a polycarbonate filter membrane. Cells were incubated at $37^{\circ} \mathrm{C}$ for $6 \mathrm{~h}$ (for accelerating siRNAs) or $9 \mathrm{~h}$ (for impairing siRNAs). Non-migrating cells on the inner sides of transwell culture inserts were then removed with a cotton swab, and migrated cells on the undersides of inserts were fixed with methanol for $10 \mathrm{~min}$ and stained with Mayer's haematoxylin (DakoCytomation, Glostrup, Denmark) for $20 \mathrm{~min}$. Photomicrographs of five random fields were taken (Olympus CK2; X100), and cells were then counted using a NIH image J program (NIH Image; Bethesda, MD). In brief, images were binary thresholded at $50 \%$ of the background level, and particles were then converted to a sub-threshold image area with a size $>200$ pixels, which was determined to be sufficient to identify migrated cells. Numbers of cells were counted and results were analysed statistically.

Figure 8 | Pivotal role of $\mathbf{p 5 5} \boldsymbol{\gamma}$ subunit of PI3K in Alk-mediated cell migration. (a,b) NIH3T3 fibroblast cells were used to reciprocally immunoprecipitate (IP) with anti-ALK and anti-PI3K antibodies as indicated. The precipitated protein was separated on SDS-PAGE and subjected to reciprocal western blot analysis (WB) using each antibody. (c) NIH3T3 fibroblast cells were transiently transfected with control vector (pcDNA3) or alk expression constructs ( $p c D N A 3-a l k)$. After $48 \mathrm{~h}$, levels of phosphorylated or total ALK, p55 $\gamma, \mathrm{p} 85 \alpha$ and AKT were evaluated by western blotting. Tubulin was used as a loading control. The results of densitometric analysis (right) are mean \pm s.d. $(n=3)$; ${ }^{\star} P$-values of $<0.05$ indicate significantly different from control vectortransfected cells. (d) NIH3T3 fibroblast cells on coverslips were transiently transfected with GFP-akt-PH expression construct and control vector ( $p c D N A 3$ ) or alk expression constructs ( $p c D N A 3-a l k$ ). After $36 \mathrm{~h}$, transfected NIH3T3 fibroblast cell images were obtained using fluorescence microscopy to determine the localization of the GFP-AKT-PH. Arrowheads indicate plasma membrane-localized GFP-AKT-PH. Scale bar, $20 \mu \mathrm{m}$. (e) ALK-overexpressing NIH3T3 fibroblast cells were transiently transfected with control siRNA or siRNAs against $p 55 \%$. After $48 \mathrm{~h}$, levels of total or phosphorylated AKT, ALK and p55 $\gamma$ were evaluated by western blotting. Tubulin was used as a loading control. The results of phospho-AKT densitometry (right) are means $\pm \mathrm{s} . \mathrm{d}$. $(n=3)$. (f) After $24 \mathrm{~h}$ of transfection, NIH3T3 fibroblast cells $\left(4 \times 10^{4}\right.$ cells/well) were seeded onto the transwell culture inserts and incubated at $37^{\circ} \mathrm{C}$ for $9 \mathrm{~h}$. The cells that migrated across the membrane were stained and counted as described in the main text. The results shown are means $\pm s . d$. $(n=3) ;{ }^{*} P$-values of $<0.05$ indicate significantly different from empty vector-transfected cells. ${ }^{\#} P$-values of $<0.05$ indicate significantly different from control siRNAtransfected cells. 
Western blot analysis. Cells were lysed in triple-detergent lysis buffer $(50 \mathrm{mM}$ Tris- $\mathrm{HCl}, \mathrm{pH} 8.0,150 \mathrm{mM} \mathrm{NaCl}, 0.02 \%$ sodium azide, $0.1 \%$ SDS, $1 \% \mathrm{NP}-40,0.5 \%$ sodium deoxycholate and $1 \mathrm{mM}$ phenylmethylsulfonyl fluoride). Protein concentrations in cell lysates were determined using the Bio-Rad protein assay kit (BioRad, Hercules, CA). Equal amounts of protein were separated by 8 or $12 \%$ SDSPAGE and transferred to Hybond ECL nitrocellulose membranes (Amersham Biosciences, Piscataway, NJ). Membranes were blocked with 5\% skim milk and sequentially incubated with primary antibodies (rabbit polyclonal anti-phosphoPI3 kinase p85 $\alpha$ (Tyr458)/p55 $\gamma$ (Tyr199) antibody (1:500 dilution; Cell Signaling Technology, Danvers, MA); rabbit polyclonal anti-PI3 kinase p85 $\alpha$ antibody (1:1,000 dilution; Cell Signaling Technology); goat polyclonal anti-p55 $\gamma$ antibody (1:500 dilution; Santa Cruz, Santa Cruz, CA); rabbit polyclonal anti-phospho-AKT (Ser473) antibody (1:1,000 dilution; Cell Signaling Technology); rabbit polyclonal anti-AKT antibody (1:1,000 dilution; Cell Signaling Technology); rabbit polyclonal anti-phospho-FOXO1 (Ser256) antibody (1:500 dilution; Cell Signaling Technology); rabbit polyclonal anti-FOXO1 antibody (1:500 dilution; Cell Signaling Technology); rabbit polyclonal anti-phospho-p70S6 kinase (Thr389) antibody (1:1,000 dilution; Cell Signaling Technology); rabbit polyclonal anti-p70S6 kinase antibody (1:1,000 dilution; Cell signaling Technology); monoclonal anti- $\alpha$-tubulin antibody (1:2,000 dilution; Sigma-Aldrich); rabbit polyclonal anti-MTMR1 antibody (1:500 dilution; Sigma-Aldrich); goat polyclonal anti-PTPN14 antibody (1:500 dilution; Santa Cruz); rabbit polyclonal anti-DOCK3 antibody (1:500 dilution; Abcam, Cambridge, MA); rabbit polyclonal anti-IRF4 antibody (1:500 dilution; Cell Signaling Technology); rabbit polyclonal anti-ALK antibody (1:500 dilution; Novus Biologicals, and HRP-conjugated secondary antibodies (1:10,000 dilution; anti-rabbit-, anti-mouse-, or anti-goat-IgG antibody; Amersham Biosciences), and then detected using an ECL detection kit (Amersham Biosciences).

Assessment of cell proliferation and viability by MTT assay. NIH3T3 cells in 96-well culture plates were transfected with siRNAs or cDNA constructs and incubated for $24-72 \mathrm{~h}$. At each designated time point after transfection, culture media were removed, MTT (3-[4,5-dimethylthiazol-2-yl]-2,5-diphenyltetrazolium bromide) $\left(0.5 \mathrm{mg} \mathrm{ml}^{-1}\right.$ was added and cells were incubated at $37^{\circ} \mathrm{C}$ for $2 \mathrm{~h}$ in a $\mathrm{CO}_{2}$ incubator. After dissolving the insoluble crystals that formed in DMSO, absorbance was measured at $570 \mathrm{~nm}$ using a microplate reader (Anthos Labtec Instruments, Wals, Austria).

Statistical analysis. Results are presented as the means \pm s.d. of three or more independent experiments, unless stated otherwise. The one-way ANOVA with Dunnett's multiple-comparison test was used to compare treatments. SPSS version $18.0 \mathrm{~K}$ (SPSS, Chicago, IL) was used for the analysis, and $P$-value differences of $<0.05$ were considered statistically significant.

\section{References}

1. Vicente-Manzanares, M., Webb, D. J. \& Horwitz, A. R. Cell migration at a glance. J. Cell. Sci. 118, 4917-4919 (2005).

2. Franz, C. M., Jones, G. E. \& Ridley, A. J. Cell migration in development and disease. Dev. Cell 2, 153-158 (2002).

3. Luster, A. D., Alon, R. \& von Andrian, U. H. Immune cell migration in inflammation: present and future therapeutic targets. Nat. Immunol. 6, 1182-1190 (2005)

4. Cram, E. J., Shang, H. \& Schwarzbauer, J. E. A systematic RNA interference screen reveals a cell migration gene network in C. elegans. J. Cell Sci. 119, 4811-4818 (2006).

5. Wang, X. et al. Analysis of cell migration using whole-genome expression profiling of migratory cells in the Drosophila ovary. Dev. Cell 10, 483-495 (2006).

6. Dykxhoorn, D. M., Novina, C. D. \& Sharp, P. A. Killing the messenger: short RNAs that silence gene expression. Nat. Rev. Mol. Cell Biol. 4, 457-467 (2003).

7. Paddison, P. J. et al. A resource for large-scale RNA-interference-based screens in mammals. Nature 428, 427-431 (2004).

8. Simpson, K. J. et al. Identification of genes that regulate epithelial cell migration using an siRNA screening approach. Nat. Cell Biol. 10, 1027-1038 (2008).

9. Vitorino, P. \& Meyer, T. Modular control of endothelial sheet migration. Gene Dev. 22, 3268-3281 (2008).

10. Winograd-Katz, S. E., Itzkovitz, S., Kam, Z. \& Geiger, B. Multiparametric analysis of focal adhesion formation by RNAi-mediated gene knockdown. J. Cell Biol. 186, 423-436 (2009).

11. Collins, C. S. et al. A small interfering RNA screen for modulators of tumor cell motility identifies MAP4K4 as a promigratory kinase. Proc. Natl Acad. Sci. USA 103, 3775-3780 (2006).

12. Bai, S. W. et al. Identification and characterization of a set of conserved and new regulators of cytoskeletal organization, cell morphology and migration. BMC Biol. 9, 54-71 (2011).

13. Smolen, G. A. et al. A genome-wide RNAi screen identifies multiple RSK-dependent regulators of cell migration. Gene Dev. 24, 2654-2665 (2010).

14. Schlabach, M. R. et al. Cancer proliferation gene discovery through functional genomics. Science 319, 620-624 (2008).
15. Silva, J. M. et al. Second-generation shRNA libraries covering the mouse and human genomes. Nat. Genet. 37, 1281-1288 (2005).

16. Yang, J. et al. Genome-wide RNAi screening identifies genes inhibiting the migration of glioblastoma cells. PLoS ONE 8, e61915 (2013).

17. Qian, Y. et al. PI3K induced actin filament remodeling through Akt and p70S6K1: implication of essential role in cell migration. Am. J. Physiol. Cell Physiol. 286, C153-C163 (2004).

18. Song, M. S., Salmena, L. \& Pandolfi, P. P. The functions and regulation of the PTEN tumour suppressor. Nat. Rev. Mol. Cell Biol. 13, 283-296 (2012).

19. Martelli, A. M. et al. Targeting the translational apparatus to improve leukemia therapy: roles of the PI3K/PTEN/Akt/mTOR pathway. Leukemia 25, 1064-1079 (2011).

20. Schlessinger, J. \& Ullrich, A. Growth factor signaling by receptor tyrosine kinases. Neuron 9, 383-391 (1992).

21. Raucher, D. et al. Phosphatidylinositol 4,5-bisphosphate functions as a second messenger that regulates cytoskeleton-plasma membrane adhesion. Cell 100, 221-228 (2000).

22. Zhu, Q. et al. Phosphoinositide 3-OH kinase p85alpha and p110beta are essential for androgen receptor transactivation and tumor progression in prostate cancers. Oncogene 27, 4569-4579 (2008).

23. Kim, D. H. \& Rossi, J. J. Strategies for silencing human disease using RNA interference. Nat. Rev. Genet. 8, 173-184 (2007).

24. Cheng, J. C., Moore, T. B. \& Sakamoto, K. M. RNA interference and human disease. Mol. Genet. Metab. 80, 121-128 (2003).

25. Alvarez-Calderon, F., Gregory, M. A. \& Degregori, J. Using functional genomics to overcome therapeutic resistance in hematological malignancies. Immunol. Res. 33, 100-115 (2012).

26. Dahlman, K. B. et al. Modulators of prostate cancer cell proliferation and viability identified by short-hairpin RNA library screening. PLoS ONE 7, e34414 (2012).

27. Hu, K., Law, J. H., Fotovati, A. \& Dunn, S. E. Small interfering RNA library screen identified polo-like kinase-1 (PLK1) as a potential therapeutic target for breast cancer that uniquely eliminates tumor-initiating cells. Breast Cancer Res. 14, R22 (2012).

28. Wan, X. et al. Identification of the FoxM1/Bub1b signaling pathway as a required component for growth and survival of rhabdomyosarcoma. Cancer Res. 72, 5889-5899 (2012).

29. Draviam, V. M. et al. A functional genomic screen identifies a role for TAO1 kinase in spindle-checkpoint signalling. Nat. Cell Biol. 9, 556-564 (2007).

30. Root, D. E., Hacohen, N., Hahn, W. C., Lander, E. S. \& Sabatini, D. M. Genome-scale loss-of-function screening with a lentiviral RNAi library. Nat. Methods 3, 715-719 (2006).

31. $\mathrm{Hu}, \mathrm{G}$. et al. A genome-wide RNAi screen identifies a new transcriptional module required for self-renewal. Gene Dev. 23, 837-848 (2009).

32. Schmitz, M. H. et al. Live-cell imaging RNAi screen identifies PP2A-B55alpha and importin-betal as key mitotic exit regulators in human cells. Nat. Cell. Biol. 12, 886-893 (2010).

33. Yang, R. et al. A genome-wide siRNA screen to identify modulators of insulin sensitivity and gluconeogenesis. PLoS ONE 7, e36384 (2012).

34. Romieu-Mourez, R., Landesman-Bollag, E., Seldin, D. C. \& Sonenshein, G. E. Protein kinase CK2 promotes aberrant activation of nuclear factor-kappaB, transformed phenotype, and survival of breast cancer cells. Cancer Res. 62, 6770-6778 (2002).

35. Channavajhala, P. \& Seldin, D. C. Functional interaction of protein kinase CK2 and c-Myc in lymphomagenesis. Oncogene 21, 5280-5288 (2002).

36. Munstermann, U. et al. Casein kinase II is elevated in solid human tumours and rapidly proliferating non-neoplastic tissue. Eur. J. Biochem. 189, 251-257 (1990).

37. Zhang, P. et al. Identification and functional characterization of p130Cas as a substrate of protein tyrosine phosphatase nonreceptor 14. Oncogene 32, 2087-2095 (2012).

38. Buj-Bello, A. et al. Muscle-specific alternative splicing of myotubularin-related 1 gene is impaired in DM1 muscle cells. Hum. Mol. Genet. 11, 2297-2307 (2002).

39. Tronchere, H. et al. Production of phosphatidylinositol 5-phosphate by the phosphoinositide 3-phosphatase myotubularin in mammalian cells. J. Biol. Chem. 279, 7304-7312 (2004).

40. Ryeom, S. et al. Targeted deletion of the calcineurin inhibitor DSCR1 suppresses tumor growth. Cancer Cell 13, 420-431 (2008).

41. Aylon, Y. et al. A positive feedback loop between the p53 and Lats2 tumor suppressors prevents tetraploidization. Genes Dev. 20, 2687-2700 (2006).

42. McPherson, J. P. et al. Lats $2 / \mathrm{Kpm}$ is required for embryonic development, proliferation control and genomic integrity. EMBO J. 23, 3677-3688 (2004).

43. Ke, H. et al. Putative tumor suppressor Lats2 induces apoptosis through downregulation of Bcl-2 and Bcl-x(L). Exp. Cell. Res. 298, 329-338 (2004).

44. Li, Y. et al. Lats2, a putative tumor suppressor, inhibits G1/S transition. Oncogene 22, 4398-4405 (2003). 
45. Huang, H. \& Tindall, D. J. Dynamic FoxO transcription factors. J. Cell Sci. 120, 2479-2487 (2007).

46. Zhang, H. et al. FOXO1 inhibits Runx2 transcriptional activity and prostate cancer cell migration and invasion. Cancer Res. 71, 3257-3267 (2011).

47. Huang, H. \& Tindall, D. J. Regulation of FOXO protein stability via ubiquitination and proteasome degradation. Biochim. Biophys. Acta 1813, 1961-1964 (2011).

48. Brunet, A. et al. Akt promotes cell survival by phosphorylating and inhibiting a Forkhead transcription factor. Cell 96, 857-868 (1999).

49. Meng, Q., Xia, C., Fang, J., Rojanasakul, Y. \& Jiang, B. H. Role of PI3K and AKT specific isoforms in ovarian cancer cell migration, invasion and proliferation through the p70S6K1 pathway. Cell Signal. 18, 2262-2271 (2006).

50. Liu, L. et al. Rapamycin inhibits cell motility by suppression of mTORmediated S6K1 and 4E-BP1 pathways. Oncogene 25, 7029-7040 (2006).

51. Poon, M. et al. Rapamycin inhibits vascular smooth muscle cell migration. J. Clin. Invest. 98, 2277-2283 (1996).

52. Bunney, T. D. \& Katan, M. Phosphoinositide signalling in cancer: beyond PI3K and PTEN. Nat. Rev. Cancer 10, 342-352 (2010).

53. Inukai, K. et al. Five isoforms of the phosphatidylinositol 3-kinase regulatory subunit exhibit different associations with receptor tyrosine kinases and their tyrosine phosphorylations. FEBS Lett. 490, 32-38 (2001).

54. Vanhaesebroeck, B., Guillermet-Guibert, J., Graupera, M. \& Bilanges, B. The emerging mechanisms of isoform-specific PI3K signalling. Nat. Rev. Mol. Cell Biol. 11, 329-341 (2010).

55. Courtney, K. D., Corcoran, R. B. \& Engelman, J. A. The PI3K pathway as drug target in human cancer. J. Clin. Oncol. 28, 1075-1083 (2010).

56 . $\mathrm{Hu}$, J. et al. Overexpression of the $\mathrm{N}$-terminal end of the p55gamma regulatory subunit of phosphatidylinositol 3-kinase blocks cell cycle progression in gastric carcinoma cells. Int. J. Oncol. 26, 1321-1327 (2005).

57. Wang, G. et al. PI3K stimulates DNA synthesis and cell-cycle progression via its p55PIK regulatory subunit interaction with PCNA. Mol. Cancer Ther. 12, 2100-2109 (2013)

58. Wang, G. et al. Blocking p55PIK signaling inhibits proliferation and induces differentiation of leukemia cells. Cell Death Differ. 19, 1870-1879 (2012).

59. Wang, G. et al. p55PIK-PI3K stimulates angiogenesis in colorectal cancer cell by activating NF-kappaB pathway. Angiogenesis 16, 561-573 (2013).

60. Zhang, L. et al. Integrative genomic analysis of phosphatidylinositol $3^{\prime}$-kinase family identifies PIK3R3 as a potential therapeutic target in epithelial ovarian cancer. Clin. Cancer Res. 13, 5314-5321 (2007).

61. Waite, K. \& Eickholt, B. J. The neurodevelopmental implications of PI3K signaling. Curr. Top. Microbiol. Immunol. 346, 245-265 (2010).

62. Roskoski, Jr. R. Anaplastic lymphoma kinase (ALK): structure, oncogenic activation, and pharmacological inhibition. Pharmacol. Res. 68, 68-94 (2013).

63. Polgar, D. et al. Truncated ALK derived from chromosomal translocation $\mathrm{t}(2 ; 5)(\mathrm{p} 23 ; \mathrm{q} 35)$ binds to the SH3 domain of p85-PI3K. Mutat. Res. 570, 9-15 (2005).
64. Wasik, M. A. et al. Anaplastic lymphoma kinase (ALK)-induced malignancies: novel mechanisms of cell transformation and potential therapeutic approaches. Semin. Oncol. 36, S27-S35 (2009).

65. Palmer, R. H., Vernersson, E., Grabbe, C. \& Hallberg, B. Anaplastic lymphoma kinase: signalling in development and disease. Biochem. J. 420, 345-361 (2009).

66. Iwahara, T. et al. Molecular characterization of ALK, a receptor tyrosine kinase expressed specifically in the nervous system. Oncogene 14, 439-449 (1997).

67. Yao, S. et al. Anaplastic lymphoma kinase is required for neurogenesis in the developing central nervous system of zebrafish. PLoS ONE 8, e63757 (2013).

68. Serrano, M., Lin, A. W., McCurrach, M. E., Beach, D. \& Lowe, S. W. Oncogenic ras provokes premature cell senescence associated with accumulation of p53 and p16INK4a. Cell 88, 593-602 (1997).

69. Seo, M., Lee, W. H. \& Suk, K. Identification of novel cell migration-promoting genes by a functional genetic screen. FASEB J. 24, 464-478 (2010).

70. Bassi, R. et al. HMGB1 as an autocrine stimulus in human T98G glioblastoma cells: role in cell growth and migration. J. Neurooncol. 87, 23-33 (2008).

\section{Acknowledgements}

This work was supported by the National Research Foundation of Korea (NRF) grant funded by the Korea government (MSIP) (Nos 2008-0062282 and 2012M3A9B6055414) and by a grant from the Korean Health technology R\&D Project, Ministry of Health \& Welfare, Republic of Korea (A111345). This work was also supported by a DOD Breast Cancer Innovator Award and a grant from SU2C to S.J.E. at the Howard Hughes Medical Institute. The authors thank Dr Young-il Park at Young-il Sciences Inc. (Daegu, Korea) for generously providing Incucyte and Woundmaker (Essen BioScience, Ann Arbor, MI).

\section{Author contributions}

M.S. and S.L. performed the experiments, analysed the data and prepared the manuscript. J.-H.K. performed the biochemical assays. W.-H.L. analysed the data. G.H. and S.J.E. provided reagents and analysed the data. K.S. directed the study and were involved in all aspects of the experimental design, data analysis and manuscript preparation. All authors critically reviewed the text and figures.

\section{Additional information}

Supplementary Information accompanies this paper at http://www.nature.com/ naturecommunications

Competing financial interests: The authors declare no competing financial interests

Reprints and permission information is available online at http://npg.nature.com/ reprintsandpermissions/

How to cite this article: Seo, M. et al. RNAi-based functional selection identifies novel cell migration determinants dependent on PI3K and AKT pathways. Nat. Commun. 5:5217 doi: 10.1038/ncomms6217 (2014). 